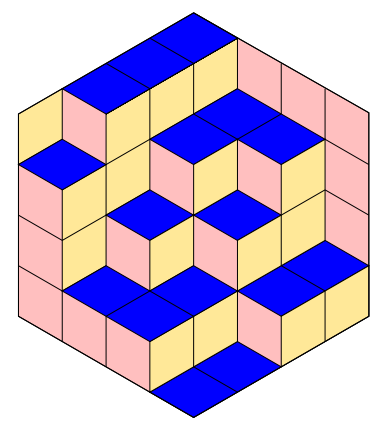

ALGEBRAIC COMBINATORICS

Mahir Bilen Can \& Jeff Remmel

Loop-augmented Forests and a Variant of Foulkes's Conjecture

Volume 1, issue 5 (2018), p. 573-601.

<http://alco.centre-mersenne.org/item/ALCO_2018__1_5_573_0>

(C) The journal and the authors, 2018.

Some rights reserved.

(c) BY This article is licensed under the

Creative Commons ATtribution 4.0 InTERnational License.

http://creativecommons.org/licenses/by/4.0/

Access to articles published by the journal Algebraic Combinatorics on the website http://alco.centre-mersenne.org/ implies agreement with the Terms of Use (http://alco.centre-mersenne.org/legal/).

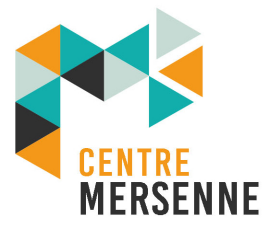

Algebraic Combinatorics is member of the Centre Mersenne for Open Scientific Publishing www.centre-mersenne.org 


\title{
Loop-augmented Forests and a Variant of Foulkes's Conjecture
}

\author{
Mahir Bilen Can \& Jeff Remmel ${ }^{\dagger}$
}

\begin{abstract}
A loop-augmented forest is a labeled rooted forest with loops on some of its roots. By exploiting an interplay between nilpotent partial functions and labeled rooted forests, we investigate the permutation action of the symmetric group on loop-augmented forests. Furthermore, we describe an extension of Foulkes's conjecture and prove a special case. Among other important outcomes of our analysis are a complete description of the stabilizer subgroup of an idempotent in the semigroup of partial transformations and a generalization of the (KnuthSagan) hook length formula.
\end{abstract}

\section{INTRODUCTION}

This paper is a natural continuation of our earlier work [1], where we considered a permutation action of the symmetric group on the set of labeled rooted forests. The origin of this representation goes back to the semigroup of partial transformations on a finite set. We shall show in this paper that the circle of ideas here naturally leads to a new variant of a long standing conjecture in representation theory, namely Foulkes's conjecture. Let us recall this famous conjecture. If $m \leqslant n$, then the multiplicity of an irreducible representation $V$ of $\mathcal{S}_{m n}$ in the induced trivial representation $\operatorname{Ind}_{\mathcal{S}_{m n} \mathcal{S}_{n}} 1$ is less than or equal to the multiplicity of $V$ in $\operatorname{Ind}_{\mathcal{S}_{n} \geq \mathcal{S}_{m}}^{\mathcal{S}_{m n}} 1$. We state this conjecture in a combinatorial way, which can be viewed as a precursor of our work.

Consider a set of vertices with $m n$ elements which are placed along a line and grouped into blocks of $m$ elements so that there are $n$ blocks in total. We will distribute the labels $1,2, \ldots, m n$ on these vertices and call two resulting distributions equivalent if one configuration can be obtained from the other by permuting the labels within the same group and/or by permuting the blocks. For example, the three configurations in the first line of Figure 1, where $n=3$ and $m=2$, are in the same equivalence class. (For convenience we separated individual blocks in each configuration by putting a bar between them.) In the second line of Figure 1, we have two inequivalent configurations since the set of elements of the first block of the first configuration is not equal to any set of elements of any blocks in the second configuration.

Manuscript received 9th August 2017, revised 1st March 2018, accepted 7th May 2018.

KEYWORDS. Labeled rooted forests, symmetric group, plethysm.

ACKnowledgements. The first author was partially supported by a grant from the Louisiana Board of Regents.

$\dagger$ Died on 29th September 2017. 


\section{(1) (2) (3) (4) (5) (6) (3) (1) (2)।(5) (6) (4) (5) (4) (6) (1) (3) (2)}

\section{(6) (2) (3) (4) (5) (1) (5) (4) (6)। (1) (3) (2)}

Figure 1. Three equivalent (in the first line), two inequivalent (in the second line) configurations.

Let us denote by $o_{1}^{\prime}$ the vector space spanned by all such equivalence classes. Next, we define another vector space, denoted by $o_{1}$, by changing the roles of $m$ and $n$. Clearly both of $o_{1}$ and $o_{1}^{\prime}$ are $\mathcal{S}_{m n}$-modules. In this terminology, Foulkes's conjecture states that the multiplicity of an irreducible $\mathcal{S}_{m n}$-module $V$ in $o_{1}^{\prime}$ is less than or equal to the multiplicity of $V$ in $o_{1}$.

Our conjecture is the following natural extension of Foulkes's conjecture. Let $m$ and $n$ be positive integers such that $n \geqslant m \geqslant 2$. Consider $m$ copies of the labeled rooted tree on $n$ vertices where the root has exactly $n-1$ children. See Figure 2, where $n=4$ and $m=2$.
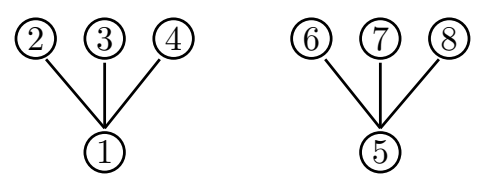

Figure 2. An extension of Foulkes's conjecture, combinatorially.

The symmetric group $\mathcal{S}_{m n}$ acts on the labels of the vertices, hence we get a representation denoted by $o_{2}^{\prime}$. In a similar manner, next, we consider $n$ copies of labeled rooted tree on $m$ vertices where the root has exactly $m-1$ children. This time the $\mathcal{S}_{m n}$ representation is denoted by $o_{2}$. Now, let $V=V(\lambda)$ be an irreducible representation of $\mathcal{S}_{m n}$ indexed by a partition $\lambda$ which has more than two parts. Then the multiplicity of $V$ in $o_{2}$ is greater than or equal to the multiplicity of $V$ in $o_{2}^{\prime}$. One of the main results of our paper is the proof of this conjecture when $m=2$.

As we mentioned before, we arrived at this conjecture by considering nilpotent partial transformations and rook theory. Our goal here is to further develop this topic, so we start with recalling the relevant concepts and results from [1]. This way, we hope that the reader will see a coherent picture of our work and the origins of our conjecture.

Let $n$ denote a positive integer, $[n]$ and $\overline{[n]}$ denote the sets $\{1, \ldots, n\}$ and $[n] \cup\{0\}$, respectively. We set

$\mathcal{F}$ ull $_{n}$ : the full transformation semigroup on $\overline{[n]}$;

$\mathcal{P}_{n}$ : the semigroup of partial transformations on $[n]$;

$\mathcal{R}_{n}$ : the rook monoid on $[n]$;

$\mathcal{C}_{n}$ : the set of nilpotent partial transformations on $[n]$.

For us, a partial transformation on $[n]$ is a function $f: A \rightarrow[n]$, where $A$ is a nonempty subset of $[n]$. A full transformation on $\overline{[n]}$ is a function $g: \overline{[n]} \rightarrow \overline{[n]}$. We note that there is an "extension by 0 " morphism from partial transformations on $[n]$ 
into full transformations on $\overline{[n]}$,

$$
\begin{aligned}
\varphi_{0}: \mathcal{P}_{n} & \longrightarrow{\mathcal{F} u l l_{n}} \\
f & \longmapsto \varphi_{0}(f)
\end{aligned}
$$

which is defined by

$$
\varphi_{0}(f)(i)= \begin{cases}f(i) & \text { if } i \text { is in the domain of } f \\ 0 & \text { otherwise. }\end{cases}
$$

The map $\varphi$ is an injective semigroup homomorphism.

By the rook monoid $\mathcal{R}_{n}$, we mean the monoid of injective partial transformations. We will often refer to the elements of $\mathcal{R}_{n}$ as rook placements (on an $n \times n$ grid). Since $\mathcal{P}_{n}$ is embedded into $\mathcal{F} u l l_{n}$ and since the latter monoid contains a zero transformation it makes sense to talk about nilpotent partial transformations in $\mathcal{P}_{n}$. In particular, $\mathcal{C}_{n}$ and $\mathcal{C}_{n} \cap \mathcal{R}_{n}$ are well defined although their definitions require an embedding of $\mathcal{P}_{n}$ into $\mathcal{F}$ ull $_{n}$. (In literature, many authors assume that $0 \in \mathcal{R}_{n}$.)

The unit groups of $\mathcal{P}_{n}$ and $\mathcal{R}_{n}$ are the same and are equal to $\mathcal{S}_{n}$. Moreover, the conjugation action of $\mathcal{S}_{n}$ on itself extends to these semigroups as well as to the subset $\mathcal{C}_{n}$ of nilpotent partial transformations. Therefore we have $\mathcal{S}_{n}$-representations on the vector spaces $\mathbb{C} \mathcal{P}_{n}, \mathbb{C} \mathcal{R}_{n}$ and on $\mathbb{C} \mathcal{C}_{n}$. The combinatorial significance of $\mathcal{C}_{n}$, which is crucial to our purposes, stems from the fact that there is a bijection between $\mathcal{C}_{n}$ and the set of labeled rooted forests on $n$ vertices. Furthermore, the conjugation action of $\mathcal{S}_{n}$ on functions in $\mathcal{C}_{n}$ translates to the permutation action of $\mathcal{S}_{n}$ on the labels.

In [1], the first author analyzed the conjugation action of $\mathcal{S}_{n}$ on $\mathcal{C}_{n}$ in detail. Our purpose here is to extend this analysis to the conjugation action of $\mathcal{S}_{n}$ on $\mathcal{P}_{n}$. By explicitly computing their stabilizer subgroups, we achieve an understanding of the orbits as permutation representations. We compute the dimensions of the corresponding representations and we determine their characters. As in our previous work, our results here are combinatorial in nature; we interpret our findings in terms of loopaugmented rooted forests (to be defined in the sequel) and plethysm operations on the symmetric functions. In the next few paragraphs we summarize the main results of [1] to set up the notation and to motivate the subsequent discussion. We should mention that we do not use rooted forests in the proofs of our results, nevertheless, as we will demonstrate in the sequel, they appear as indispensable tools for our Frobenius character calculus. Of course, as we showed at the beginning of our article, they are proven to be useful for paraphrasing (and extending) Foulkes's conjecture.

A pair $(\tau, \phi)$, where $\tau$ is a rooted forest on $n$ vertices and $\phi$ is a bijective map from $[n]$ onto the vertex set of $\tau$ is called a labeled rooted forest. Throughout this paper, when we talk about labeled rooted forests, we omit writing the corresponding labeling function despite the fact that the action of $\mathcal{S}_{n}$ does not change the underlying forest but the labeling function only. In accordance with this convention, when we write $\mathcal{S}_{n} \cdot \tau$ we actually mean the orbit

$$
\mathcal{S}_{n} \cdot(\tau, \phi)=\left\{\left(\tau, \phi^{\prime}\right): \phi^{\prime}=\sigma \cdot \phi, \sigma \in \mathcal{S}_{n}\right\} .
$$

The right hand side of (1) is an $\mathcal{S}_{n}$-set, hence it defines a representation of $\mathcal{S}_{n}$. We call the resulting representation the odun of $\tau$ and denote it by $o(\tau)$. It only depends on $\tau$, so we write $\operatorname{Stab}_{\mathcal{S}_{n}}(\tau)$ to denote the stabilizer subgroup of the pair $(\tau, \phi)$. As an $\mathcal{S}_{n}$-module, the vector space of functions on the right cosets, that is $\mathbb{C}\left[\mathcal{S}_{n} / \operatorname{Stab}_{\mathcal{S}_{n}}(\tau)\right]$ is isomorphic to the odun of $\tau$, which is equivalent to the induced representation $\operatorname{Ind}_{\operatorname{Stab}_{\mathcal{S}_{n}}(\tau)}^{\mathcal{S}_{n}} \mathbb{1}$. It turns out that the Frobenius character of this module is recursive in nature and can be computed by certain combinatorial rules. To avoid introducing more notation, we illustrate these rules on an example. Let $\tau$ be the rooted forest 
depicted in Figure 3 and let $\tau_{i}, i=1,2,3$ denote its connected components (from left to right in the figure).

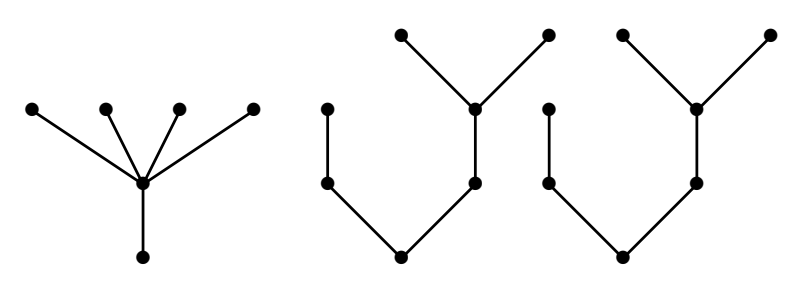

Figure 3. An example.

Let $F_{\tau}$ and $F_{\tau_{i}}, i=1,2,3$ denote the corresponding Frobenius characters. Since $\tau_{1} \neq \tau_{2}=\tau_{3}$, we have

$$
F_{\tau}=F_{\tau_{1}} \cdot s_{2}\left[F_{\tau_{2}}\right]
$$

Here, $s_{2}$ is the Schur function $s_{(2)}$, the bracket stands for the plethysm of symmetric functions and the dot stands for ordinary multiplication. (We will give a brief introduction to plethysm in Section 2.2.) More generally, if a connected component $\tau^{\prime}$, which of course is a rooted tree appears $k$-times in a forest $\tau$, then $F_{\tau}$ has $s_{k}\left[F_{\tau^{\prime}}\right]$ as a factor. Now we proceed to explain the computation of the Frobenius character of a rooted tree. As an example we use $F_{\tau_{1}}$ of Figure 3 . The combinatorial rule that we obtained in [1] is simple; it is the removal of the root from the tree. The effect on the Frobenius character of this simple rule is as follows: let $\tau_{1}^{\prime}$ denote the rooted forest that we obtain from $\tau_{1}$ by removing the root. Then $F_{\tau_{1}}=s_{1} \cdot F_{\tau_{1}^{\prime}}$. Thus, by the repeated application of this rule and the previous factorization rule, we obtain $F_{\tau_{1}}=s_{1} \cdot F_{\tau_{1}^{\prime}}=s_{1} \cdot s_{1} \cdot s_{4}\left[s_{1}\right]$. It follows from the definition of plethysm that $s_{k}\left[s_{1}\right]=s_{k}$ for any nonnegative integer $k$. Therefore,

$$
F_{\tau_{1}}=s_{1}^{2} \cdot s_{4}
$$

We compute $F_{\tau_{2}}$ by the same method;

$$
F_{\tau_{2}}=s_{1}^{5} \cdot s_{2}
$$

By putting (2)-(4) together, we arrive at the following satisfactory expression for the Frobenius character of $\tau$ :

$$
F_{\tau}=F_{\tau_{1}} \cdot s_{2}\left[F_{\tau_{2}}\right]=s_{1}^{2} \cdot s_{4} \cdot s_{2}\left[s_{1}^{5} \cdot s_{2}\right] .
$$

Note that the expansion of $s_{2}\left[s_{1}^{5} \cdot s_{2}\right]$ in the Schur basis is computable by a recursive method by applying (14) and using Thrall's formula [9] (see [7, Chapter I, Section 8, Example 9]). However, the resulting expression is rather large, so, we omit writing it here.

Let $\tau$ be a rooted tree with the root $c$ and let $a$ and $b$ be two vertices from $\tau$. Let $p_{a}$ (resp. $p_{b}$ ) denote the unique path between $a$ and $c$ (resp. between $b$ and $c$ ). We define a partial order, denoted by $\leqslant$, on $\tau$ by declaring $b \leqslant a$ if $a$ lies on the path $p_{b}$. This definition extends to rooted forests as follows. If $a$ and $b$ are two vertices from different connected components in a rooted forest, then they are declared to be incomparable with respect to $\leqslant$.

Let $\sigma$ be a rooted tree on $n+1$ vertices and let $a$ be a vertex in $\sigma$. We let $\sigma_{a}^{0}$ denote the rooted subforest whose set of vertices is $\{b \in \sigma: b<a\}$ and we let $\sigma_{a}$ denote the rooted subtree whose set of vertices is $\{b \in \sigma: b \leqslant a\}$. Then we denote by $\gamma_{a}$ the subset $\left\{a_{1}, \ldots, a_{r}\right\}$ of the vertices of $\sigma_{a}$ such that the corresponding subtrees $\sigma_{a_{i}}$ $(i=1, \ldots, r)$ are mutually disjoint and such that $\cup_{i=1}^{r} \sigma_{a_{i}}=\sigma_{a}^{0}$. We call each of the 
subtrees $\sigma_{a_{i}}(i=1, \ldots, r)$ a maximal branch at $a$. Finally, let $m\left(a ; a_{i}\right)=m_{\sigma}\left(a ; a_{i}\right)$ $(i=1, \ldots, r)$ denote the number of copies of $\sigma_{a_{i}}$ that appear in $\sigma_{a}^{0}$. Let us call the number $m\left(a ; a_{i}\right)$ the multiplicity of $a$ at $a_{i}$. In this notation, the dimension of the odun of $\sigma$ turns out to be

$$
\operatorname{dim}_{\mathbb{C}} O(\sigma)=\frac{n !}{\prod_{a \in \sigma} \prod_{b \in \gamma_{a}} m(a ; b) !},
$$

where it is understood that the multiplicity $m(a ; b)$ is used once for all isomorphic copies of $\sigma_{b}$ in $\sigma_{a}^{0}$. The same formula applies to the dimension of the odun of the rooted forest $\tau$ that is obtained from $\sigma$ by removing its root (see [1, Theorem 9.3]). This is a generalization of the Knuth-Sagan hook length formula for the rooted trees.

Let us demonstrate our formula (6) on the rooted tree, denoted by $\sigma$, that is obtained from the rooted forest $\tau$ in Figure 3 by adding a vertex that serves the purpose of a root. Let us denote this root by $c$. Note that $n+1=21$. We name the vertices of the leftmost tree in Figure 3 by $a_{1}, \ldots, a_{6}$ from bottom to top and left to right. Thus, $a_{1}$ is the root. In a similar manner, we denote by $b_{1}, \ldots, b_{7}$ and $c_{1}, \ldots, c_{7}$, respectively, the vertices of the middle rooted tree and the rightmost rooted tree so that $b_{1}$ and $c_{1}$, respectively, are the corresponding roots. Then $\gamma_{c}=\left\{a_{1}, b_{1}, c_{1}\right\}$, and there is one copy of $\tau_{a_{1}}$ in $\sigma$ and there are two copies of the subtree $\tau_{b_{1}}$ in $\sigma$. Thus $m\left(c ; a_{1}\right)=1, m\left(c ; b_{1}\right)=2$. In a similar manner, we compute $m\left(a_{2}, a_{3}\right)=4$, $m\left(b_{5}, b_{6}\right)=2=m\left(c_{5}, c_{6}\right)$, and all other multiplicities are equal to 1 . Then

$$
\operatorname{dim}_{\mathbb{C}} o(\sigma)=\operatorname{dim}_{\mathbb{C}}(\tau)=\frac{20 !}{4 ! 2 ! 2 ! 2 !}=12671364625920000 .
$$

This number can be verified by using the Frobenius character (5) of $\tau$.

We are now ready to introduce our new combinatorial object by means of which we will generalize the results of [1]. The algebraic significance of these objects will be developed later in this paper. A loop-augmented forest is a rooted forest such that there is at most one loop at each of its roots. See, for example, Figure 4, where we depict a loop-augmented forest on 22 vertices and four loops.
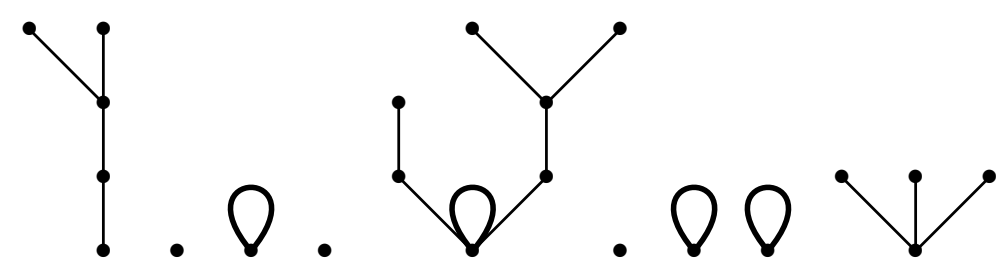

Figure 4. A loop-augmented forest.

It is a well known variation of the Cayley's theorem that the number of labeled forests on $n$ vertices with $k$ roots is equal to $\left(\begin{array}{c}n-1 \\ k-1\end{array}\right) n^{n-k}$. See [3, Theorem D, p. 70]. It follows that the number of loop-augmented forests on $n$ vertices with $k$ roots is $2^{k}\left(\begin{array}{c}n-1 \\ k-1\end{array}\right) n^{n-k}$. It follows from generating function manipulations that the number of loop-augmented forests on $n$ vertices for $n \geqslant 2$ is $2 n^{n-3}$.

Next, we explain the relationship between the rooted forests and nilpotent partial transformations. Let $\tau$ be a rooted tree on $n$ vertices. We group the maximal branches of $\tau$ according to the number of vertices on each maximal branch. As a convention, let us collect the maximal branches with more vertices on the left hand side of the graph and repeat this procedure inductively for every maximal branch and for their maximal branches, and so on. We do not worry about the ordering of the maximal branches in the same group. As an example, see Figure 5. Once the grouping is done, 
we label the vertices by $[n]$ from left to right and from bottom to top, decreasing the label by 1 each time. Here, $n$ is the number of vertices and the root of $\tau$ is labeled by $n$.

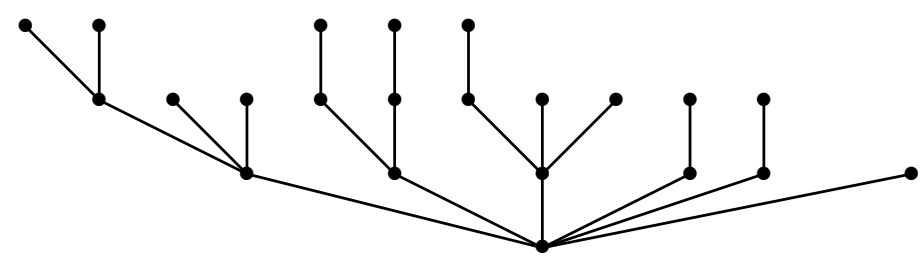

FiguRE 5. A grouping of the branches of a rooted tree.

Notwithstanding the ambiguity created by not ordering the maximal branches of the same size, the resulting incidence matrix from our labeling is a strictly upper triangular (hence, nilpotent) matrix. We adopt this procedure to the rooted forests by first grouping (and depicting left to right) the rooted trees with respect to the number of vertices they have. The resulting incidence matrix of the forest is a direct sum of nilpotent matrices, hence it is nilpotent also. Notice that this procedure also explains why there is a bijection between labeled rooted forests and nilpotent partial functions. That is, given $\tau \in \mathcal{C}_{n}$ with respect to any labeling that we just described, we simply let $f$ be the partial function defined by $f(i)=j$ whenever $i$ is a child of $j$ in $\tau$ and there is an edge between $i$ and $j$. Then $f$ is nilpotent. In the light of this fact, from now on, we will use the same notation $\tau$ for a nilpotent partial function and its corresponding labeled rooted forest.

We now proceed to explain how to interpret loop-augmented forests in terms of partial functions. Let $\sigma$ be a loop-augmented forest. Then some of the roots of $\sigma$ have loops. There is still a partial function for $\sigma$, as defined in the previous paragraph for a rooted forest. The loops in this case correspond to the "fixed points" of the associated function. Indeed, with respect to labeling conventions of the previous paragraph, the incidence matrix of $\sigma$ is upper triangular. However it can have both of 1's and 0's on its main diagonal. The resulting matrix can be viewed as the matrix representation of the partial function of $\sigma$. (Warning: unlike our notation in this paragraph, in the sequel we will often use lower case Latin letters for denoting both of loop-augmented forests and partial functions.) We remark here that the correspondence between loopaugmented forests and (certain) partial functions fails if we consider adding loops on non-roots. In such a situation, the resulting objects do not always give partial functions, but, instead, give "partial relations."

The subset of $\mathcal{P}_{n}$ which corresponds to the loop-augmented forests will be formally defined in the next section. Now, we briefly explain the underlying algebraic theme of our work. Recall that the permutation action of $\mathcal{S}_{n}$ on the labels translates to the conjugation action on the incidence matrices. Clearly, the conjugates of a nilpotent (respectively unipotent) matrix are still nilpotent (respectively unipotent). Let $U$ be an arbitrary upper triangular matrix. Then $U$ is equal to a sum of the form $D+N$, where $D$ is a diagonal matrix and $N$ is a nilpotent matrix. If $\sigma$ is a permutation matrix of the same size as $U$, then we conclude from the equalities $\sigma \cdot U=\sigma U \sigma^{-1}=\sigma D \sigma^{-1}+$ $\sigma N \sigma^{-1}$ that the conjugation action on loop-augmented forests is equivalent to the simultaneous conjugation action on labeled rooted forests and the representation of $\mathcal{S}_{n}$ on diagonal matrices.

The outline of our paper is as follows. In Section 2, we review the most basic facts about the idempotents of the semigroups $\mathcal{F} u l l_{n}, \mathcal{P}_{n}$, and $\mathcal{R}_{n}$. In addition we review 
the basic theory of plethysm for symmetric functions. In Section 3, we prove the first of our main results, Theorem 3.2, which states that the stabilizer subgroup in $\mathcal{S}_{n}$ of a loop-augmented forest (viewed as a partial function on $[n]$ ) has a direct product decomposition of the form $Z(\sigma) \times \operatorname{Stab}_{\mathcal{S}_{n-k}}(\tau)$, where $Z(\sigma)$ is the centralizer of a permutation $\sigma$ in $\mathcal{S}_{k}$ and $\operatorname{Stab}_{\mathcal{S}_{n-k}}(\tau)$ is the stabilizer subgroup in $\mathcal{S}_{n-k}$ of a labeled rooted forest $\tau$. It follows from this result that the Frobenius character of the $\mathcal{S}_{n^{-}}$ orbit of a loop-augmented forest $f$ is of the form $s_{\lambda} \prod_{i=1}^{r} h_{\lambda_{i}}\left[F_{\tau_{i}}\right]$, where $\tau_{1}, \ldots, \tau_{r}$ are the distinct connected components of $\tau$ where $\tau_{i}$ occurs $\lambda_{i}$ times. We record this theorem as Theorem 4.2 in Section 4. Another consequence of the factorization of the stabilizer subgroup is a dimension formula for the $\mathcal{S}_{n}$ representation defined by $f$ (Corollary 3.3). If $\left(1^{m_{1}} 2^{m_{2}} \ldots k^{m_{k}}\right)$ denotes the conjugacy type of $\sigma$ in $\mathcal{S}_{k}$, then the dimension of $\mathbb{C}\left[\mathcal{S}_{n} \cdot f\right]$ is given by

$$
\frac{n !}{k !} \frac{\prod_{j} j^{m_{j}}\left(m_{j} !\right)}{\left(\prod_{a \in \tau} \prod_{b \in \gamma_{a}} m(a ; b) !\right)},
$$

where $m(a ; b)=m_{\tau}(a ; b)$ is as defined in (6). In Section 5 , we prove the main algebraic result of our paper. Namely, if $e \in \mathcal{P}_{n}$ is an arbitrary idempotent, then there exists a multiset of integers $\left\{\beta_{1}^{m_{1}}, \ldots, \beta_{s}^{m_{s}}\right\}$ and $m \in \mathbb{N}$ such that

(1) $m+\sum_{i=1}^{s} m_{i} \beta_{i}=n$;

(2) $\operatorname{Stab}_{\mathcal{S}_{n}}(e) \simeq\left(\mathcal{S}_{m_{1}} \prec \mathcal{S}_{\beta_{1}}\right) \times\left(\mathcal{S}_{m_{2}} \prec \mathcal{S}_{\beta_{2}}\right) \times \cdots \times\left(\mathcal{S}_{m_{s}} \prec \mathcal{S}_{\beta_{s}}\right) \times \mathcal{S}_{m}$.

We record this result in Corollary 5.6. Finally, in Section 6, we prove our variant of Foulkes's conjecture in the special case where $m=2$. (See Conjecture 6.4 for an equivalent statement in terms of symmetric functions and plethysm.)

\section{Preliminaries}

Structures of the semigroups $\mathcal{P}_{n}$ and $\mathcal{R}_{n}$ become more transparent by virtue of matrices; each element $g$ of $\mathcal{P}_{n}$ has a unique $n \times n$ matrix representative $g=\left(g_{i j}\right)_{i, j=1}^{n}$ defined by

$$
g_{j i}=\left\{\begin{array}{ll}
1 & \text { if } g(i)=j ; \\
0 & \text { otherwise }
\end{array} \quad(i, j \in[n]) .\right.
$$

What is important for us is that the matrix product translates to the semigroup multiplication (functional composition). In particular, a partial transformation $f \in \mathcal{P}_{n}$ is nilpotent if some power of the corresponding matrix $f=\left(f_{i, j}\right)_{i, j=1}^{n}$ is the zero matrix $\mathbf{0}_{n}$.

By a rook matrix, we mean a $0 / 1$ matrix that corresponds to an injective partial transformation by the above association (8).

2.1. IDEmpotents. An idempotent in a semigroup $S$ is an element $e \in S$ satisfying $e^{2}=e$. The identity transformation $\mathrm{id}_{n}:[n] \rightarrow[n]$ of the unit group is an idempotent, and conversely for any idempotent $e \in S:=\mathcal{P}_{n}$, there is a corresponding "local group" $S_{e}$ determined by $e$; it is defined as $S_{e}:=e \mathcal{P}_{n} e$. The identity of the local group $S_{e}$ is $e$. We denote the idempotent set of a semigroup $S$ by $E(S)$.

By a diagonal idempotent in $\mathcal{P}_{n}$, we mean an idempotent of $\mathcal{R}_{n}$. The nomenclature follows from the fact that the matrix representation of $e \in E\left(\mathcal{R}_{n}\right)$ is a diagonal matrix. $E\left(\mathcal{R}_{n}\right) \subset \mathcal{R}_{n}$ has exactly $2^{n}$ elements (including the 0 -matrix) and it forms a commutative submonoid of $\mathcal{R}_{n}$. It is not difficult to see that $E\left(\mathcal{R}_{n}\right)$ is isomorphic to the "face lattice" of the regular " $n$-simplex" in $\mathbb{R}^{n}$. For example,

$$
E\left(\mathcal{R}_{2}\right)=\left\{\left(\begin{array}{ll}
1 & 0 \\
0 & 1
\end{array}\right),\left(\begin{array}{ll}
1 & 0 \\
0 & 0
\end{array}\right),\left(\begin{array}{ll}
0 & 0 \\
0 & 1
\end{array}\right),\left(\begin{array}{ll}
0 & 0 \\
0 & 0
\end{array}\right)\right\} .
$$


Let $U$ denote the unit group of a semigroup $S$. For our purposes it is important to note that $E(S)$ is closed under the conjugation action

$$
g \cdot e=g e g^{-1}, \quad g \in U, e \in E(S) .
$$

In particular we see that $E\left(\mathcal{R}_{n}\right)$ is an $\mathcal{S}_{n}$-set that is contained in $E\left(\mathcal{P}_{n}\right)$. For example,

$$
E\left(\mathcal{P}_{2}\right)=\left\{\left(\begin{array}{ll}
1 & 1 \\
0 & 0
\end{array}\right),\left(\begin{array}{ll}
0 & 0 \\
1 & 1
\end{array}\right)\right\} \bigcup E\left(\mathcal{R}_{2}\right) .
$$

We list some easy facts about the set of idempotents of $\mathcal{P}_{n}$. For the proofs of some of these facts (and more) we recommend [4].

(1) The number of idempotents in $\mathcal{P}_{n}$ is given by $\sum_{k=0}^{n}\left(\begin{array}{l}n \\ k\end{array}\right)(k+1)^{n-k}$.

(2) The number of idempotents in $\mathcal{F} u l l_{n}$ is given by $\sum_{k=1}^{n}\left(\begin{array}{l}n \\ k\end{array}\right) k^{n-k}$.

(3) The idempotents in $\mathcal{P}_{n}$ do not form a subsemigroup of $\mathcal{P}_{n}$ for $n \geqslant 2$.

(4) The idempotents in $\mathcal{F} u l l_{n}$ do not form a subsemigroup of $\mathcal{F} u l l_{n}$ for $n \geqslant 3$.

(5) The idempotents in $\mathcal{R}_{n}$ form a submonoid of $\mathcal{R}_{n}$.

Let $e$ be a diagonal idempotent in $\mathcal{P}_{n}$. Since all idempotents of rank $k$ in $\mathcal{R}_{n}$ are related to each other via conjugation, the $\mathcal{S}_{n}$-orbit of $e$ is uniquely determined by the number $k=\operatorname{rank}(e)$. We define

(9) $\mathcal{N}(n, k)=\left\{f \in \mathcal{P}_{n}: f^{m}\right.$ is a rank $k$ diagonal idempotent for some $\left.m \geqslant 1\right\}$,

which is closed under conjugation action of $\mathcal{S}_{n}$. Clearly, if $k=0$, then $\mathcal{N}(n, k)$ is equal to $\mathcal{C}_{n}$, the set of nilpotent elements in $\mathcal{P}_{n}$.

REMARK 2.1. By relaxing the requirement on diagonal property, we could have considered

$$
\left\{f \in \mathcal{P}_{n}: f^{m} \text { is a rank } k \text { idempotent for some } m \geqslant 1\right\} .
$$

This set properly contains $\mathcal{N}(n, k)$. However, the matrix rank of idempotents is not a refined invariant with respect to conjugation action. To justify this claim, we consider

$$
e_{1}:=\left(\begin{array}{ll}
1 & 0 \\
0 & 0
\end{array}\right) \text { and } e_{2}:=\left(\begin{array}{ll}
1 & 1 \\
0 & 0
\end{array}\right) .
$$

These idempotents are of the same rank but they are not conjugate. To capture differences between the conjugacy classes of $e_{1}$ and $e_{2}$, we will introduce below the concept of "standard idempotent."

Let $e \in E\left(\mathcal{P}_{n}\right)$ be an idempotent with the property that the only nonzero row of $e$ has 1's as its entries. For example, $e=\left(\begin{array}{lll}0 & 0 & 0 \\ 0 & 0 & 0 \\ 1 & 1 & 1\end{array}\right)$. Such idempotents, viewed as functions, are constant. Conversely, any nonzero constant function $f:[n] \rightarrow[n]$ defines an idempotent $e \in \mathcal{P}_{n}$ with a single row of 1's.

Definition 2.2. An idempotent is called constant, and denoted by $c^{(n)}$, if the corresponding function is 1 . In other words $c^{(n)}(i)=1$ for all $i \in\{1, \ldots, n\}$. An idempotent in $\mathcal{P}_{n}$ is called standard if it is a direct sum of constant idempotents of various sizes plus a 0-matrix.

Let $x \in \mathcal{P}_{n}$. The following set will be useful in the sequel:

$$
\mathcal{M}(n, x):=\left\{f \in \mathcal{P}_{n}: f^{m} \text { is conjugate to } x \text { for some } m \geqslant 1\right\} .
$$

LEMma 2.3. For every $x \in \mathcal{P}_{n}$, the set $\mathcal{M}(n, x)$ is closed under the conjugation action of $\mathcal{S}_{n}$. Furthermore, if $x$ is a diagonal idempotent of rank $k$, then $\mathcal{N}(n, k)=\mathcal{M}(n, x)$. 
Proof. Let $f$ be an element in $\mathcal{M}(n, x)$. By definition, there exists $y \in \mathcal{S}_{n}$ such that $y f^{m} y^{-1}=e$ for some $m \geqslant 1$. We will show that $z f z^{-1} \in \mathcal{M}(n, x)$ for any $z \in \mathcal{S}_{n}$. Indeed,

$$
y z^{-1}\left(z f z^{-1}\right)^{m}\left(y z^{-1}\right)^{-1}=y f^{m} y^{-1}=x .
$$

This proves the first claim. The proof of the second claim follows from the first claim and the fact that $\mathcal{S}_{n}$ acts transitively on the set of diagonal idempotents of fixed rank.

REMARK 2.4. The reason behind introducing the set $\mathcal{N}(n, k)$ is that it is precisely the subset of $\mathcal{P}_{n}$ that corresponds to the loop-augmented forests with $k$ loops. Thus, our main goal is to understand the $\mathcal{S}_{n}$-module structure on $\mathcal{N}(n, k)$.

2.2. Symmetric Functions AND Plethysm. In representation theory, plethysm refers to a general idea on a functorial operation, which is defined from a representation to another one. More concretely, it is a description of the decompositions, with multiplicities, of representations derived from a given representation $V$ such as $V \otimes V, \bigwedge^{k}(V), \bigwedge^{k}\left(\bigwedge^{l}(V)\right), \operatorname{Sym}^{k}(V), \operatorname{Sym}^{l}\left(\operatorname{Sym}^{k}(V)\right), V^{*} .$. In practice one often considers functors attached to the weight of an irreducible representation such as Schur functors. Let $\mathbb{S}_{\mu}$ resp. $\mathbb{S}_{\lambda}$ denote the Schur functors indexed by the partitions $\mu$ and $\lambda$, respectively. Then $\mathbb{S}_{\lambda} \circ \mathbb{S}_{\mu}:=\mathbb{S}_{\lambda}\left(\mathbb{S}_{\mu}\left(\mathbb{C}^{n}\right)\right)$ is called the plethysm of $\mathbb{S}_{\lambda}$ with $\mathbb{S}_{\mu}$. There is a corresponding operation on the ring of symmetric functions.

The $k$-th power-sum symmetric function, denoted by $p_{k}$, is the sum of $k$-th powers of the variables. For a partition $\lambda=\left(\lambda_{1} \leqslant \ldots \leqslant \lambda_{l}\right)$, we define $p_{\lambda}$ to be equal to $\prod_{i} p_{\lambda_{i}}$. (We will maintain the convention of writing parts of a partition in the increasing order throughout our paper.) The $k$-th complete symmetric function, $h_{k}$, is defined to be the sum of all monomials $x_{i_{1}}^{a_{1}} \cdots x_{i_{r}}^{a_{r}}$ with $\sum a_{i}=k$ and $h_{\lambda}$ is defined to be equal to $\prod_{i=1}^{l} h_{\lambda_{i}}$. The Schur function associated with $\lambda$, denoted by $s_{\lambda}$, is the symmetric function defined by the determinant $\operatorname{det}\left(h_{\lambda_{i}+j-i}\right)_{i, j=1}^{l}$. In particular, we have, for all $k \geqslant 1$, that $s_{(k)}=h_{k}$. For easing the notation, we denote $s_{(k)}$ by $s_{k}$. The $k$-th elementary symmetric function, denoted by $e_{k}$, can be defined as the Schur function $s_{\left(1^{k}\right)}$, where $\left(1^{k}\right)$ is the partition of $k$ whose parts are all equal to 1 .

The type of a conjugacy class $\sigma$ in $\mathcal{S}_{n}$ is the partition $\lambda$ whose parts correspond to the lengths of the cycles that appear in the cycle decomposition of an element $x \in \sigma$. It is well known that the type is independent of the element $x$, furthermore, it uniquely determines the conjugacy class.

The power sum and Schur symmetric functions will play special roles in our computations via the Frobenius character map

$$
\text { F : class functions on } \begin{aligned}
\mathcal{S}_{n} & \longrightarrow \text { symmetric functions } \\
\delta_{\sigma} & \longmapsto \frac{1}{n !} p_{\lambda},
\end{aligned}
$$

where $\sigma \subset \mathcal{S}_{n}$ is a conjugacy class of type $\lambda$ and $\delta_{\sigma}$ is the indicator function

$$
\delta_{\sigma}(x)= \begin{cases}1 & \text { if } x \in \sigma \\ 0 & \text { otherwise. }\end{cases}
$$

It turns out that if $\chi^{\lambda}$ is the irreducible character of $\mathcal{S}_{n}$ indexed by the partition $\lambda$, then $\mathbf{F}\left(\chi^{\lambda}\right)=s_{\lambda}$. In the sequel, we will not distinguish between representations of $\mathcal{S}_{n}$ and their corresponding characters. In particular, we will often write the Frobenius character of an orbit to mean the image under $\mathbf{F}$ of the character of the representation of $\mathcal{S}_{n}$ that is defined by the action on the orbit.

For two irreducible characters $\chi^{\mu}$ and $\chi^{\lambda}$ indexed by partitions $\lambda$ and $\mu$, the Frobenius character image of the plethysm $\chi^{\lambda}\left[\chi^{\mu}\right]$ is the plethystic substitution $s_{\lambda}\left[s_{\mu}\right]$ of the 
corresponding Schur functions. Roughly speaking, the plethysm of the Schur function $s_{\lambda}$ with $s_{\mu}$ is the symmetric function obtained from $s_{\lambda}$ by substituting the monomials of $s_{\mu}$ for the variables of $s_{\lambda}$. In the notation of [6]; the plethysm of symmetric functions is the unique map [.] $: \Lambda \times \Lambda \rightarrow \Lambda$ satisfying the following three axioms:

(P1) For all $m, n \geqslant 1, p_{m}\left[p_{n}\right]=p_{m n}$.

(P2) For all $m \geqslant 1$, the map $g \mapsto p_{m}[g], g \in \Lambda$ defines a $\mathbb{Q}$-algebra homomorphism on $\Lambda$.

(P3) For all $g \in \Lambda$, the map $h \mapsto h[g], h \in \Lambda$ defines a $\mathbb{Q}$-algebra homomorphism on $\Lambda$.

Although the problem of computing the plethysm of two (arbitrary) symmetric functions is very difficult, there are some useful formulas for Schur functions:

$$
s_{\lambda}[g+h]=\sum_{\mu, \nu} c_{\mu, \nu}^{\lambda}\left(s_{\mu}[g]\right)\left(s_{\nu}[h]\right),
$$

and

$$
s_{\lambda}[g h]=\sum_{\mu, \nu} \gamma_{\mu, \nu}^{\lambda}\left(s_{\mu}[g]\right)\left(s_{\nu}[h]\right) .
$$

Here, $g$ and $h$ are arbitrary symmetric functions, $c_{\mu, \nu}^{\lambda}$ is a scalar, and $\gamma_{\mu, \nu}^{\lambda}$ is $\frac{1}{n !}\left\langle\chi^{\lambda}, \chi^{\mu} \chi^{\nu}\right\rangle$, where the pairing stands for the standard Hall inner product on characters.

In (12) the summation is over all pairs of partitions $\mu, \nu \subset \lambda$, and the summation in (13) is over all pairs of partitions $\mu, \nu$ such that $|\mu|=|\nu|=|\lambda|$. In the special case when $\lambda=(n)$, or $\left(1^{n}\right)$ we have

$$
\begin{aligned}
s_{(n)}[g h] & =\sum_{\lambda \vdash n}\left(s_{\lambda}[g]\right)\left(s_{\lambda}[h]\right), \\
s_{\left(1^{n}\right)}[g h] & =\sum_{\lambda \vdash n}\left(s_{\lambda}[g]\right)\left(s_{\lambda^{\prime}}[h]\right),
\end{aligned}
$$

where $\lambda^{\prime}$ denotes the conjugate of $\lambda$.

Finally, let us mention the conjugation property of plethysm:

$$
\left\langle s_{\lambda}\left[s_{\mu}\right], s_{\gamma}\right\rangle= \begin{cases}\left\langle s_{\lambda}\left[s_{\mu^{\prime}}\right], s_{\gamma^{\prime}}\right\rangle & \text { if }|\mu| \text { is even; } \\ \left\langle s_{\lambda^{\prime}}\left[s_{\mu^{\prime}}\right], s_{\gamma^{\prime}}\right\rangle & \text { if }|\mu| \text { is odd. }\end{cases}
$$

\section{Nilpotents and Diagonal Idempotents}

Let $f$ be an element of $\mathcal{N}(n, k), m$ be a positive integer such that $f^{m}$ is a diagonal idempotent in $\mathcal{P}_{n}$. Without loss of generality we assume that $f^{m}$ is of the form

$$
f^{m}=\left(\begin{array}{cc}
\operatorname{id}_{k} & 0 \\
0 & 0
\end{array}\right)
$$

Our first result is about the actual "shape" of the matrix representation of $f$.

Proposition 3.1. If $f$ is a partial transformation as in the previous paragraph, then there exist a permutation $\sigma \in \mathcal{S}_{k}$ and a nilpotent partial transformation $\tau$ from $\mathcal{C}_{n-k}$ such that

$$
f=\left(\begin{array}{cc}
\sigma & 0 \\
0 & \tau
\end{array}\right)
$$

Proof. First, we are going to show that $f$ has the block-diagonal form as in (18). We will prove this by contradiction, so we assume that there exists $j \in\{k+1, \ldots, n\}$ such that $f(j)=i<k+1$. On one hand, since $f^{m}$ is as in (17), we have $f^{m}(i)=i$. On the other hand, by our assumption $0=f^{m}(j)=f^{m-1}(f(j))=f^{m-1}(i)$. Therefore, 
$f^{m}(i)=0$, but this is a contradiction. Therefore, $f$ is as in (18) for some $\sigma \in \mathcal{P}_{k}$ and $\tau \in \mathcal{C}_{n-k}$.

Since the $m$-th power of $\left(\begin{array}{cc}\sigma & 0 \\ 0 & \tau\end{array}\right)$ is as in (17), them-th power of $\sigma$ is the $k \times k$ identity matrix and the $m$-th power of $\tau$ is $\mathbf{0}_{n-k}$. In other words, $\sigma$ is a permutation matrix, $\tau$ is a nilpotent matrix. The proof is complete.

THEOREM 3.2. Let $f$ be a partial transformation of the form $f=\left(\begin{array}{cc}\sigma & 0 \\ 0 & \tau\end{array}\right)$, where $\sigma \in \mathcal{S}_{k}$ is a permutation and $\tau \in \mathcal{C}_{n-k}$ is a nilpotent partial transformation. In this case, the stabilizer subgroup in $\mathcal{S}_{n}$ of $f$ has the following decomposition:

$$
\operatorname{Stab}_{\mathcal{S}_{n}}(f)=Z(\sigma) \times \operatorname{Stab}_{\mathcal{S}_{n-k}}(\tau),
$$

where $Z(\sigma)$ is the centralizer of $\sigma$ in $\mathcal{S}_{k}$ and $\operatorname{Stab}_{\mathcal{S}_{n-k}}(\tau)$ is the stabilizer subgroup of $\tau$ in $\mathcal{S}_{n-k}$.

Proof. Let $\pi=\left(\begin{array}{cc}A & B \\ C\end{array}\right) \in \mathcal{S}_{n}$ be a permutation matrix given in block form so that $A$ is a $k \times k$-matrix, $D$ is $n-k \times n-k$-matrix, $B$ is $k \times n-k$-matrix, and $C$ is $n-k \times k$ matrix. Assume that $\pi f \pi^{-1}=f$. We claim that both $B$ and $C$ are 0 matrices. To see this, we use $\pi f=f \pi$ to get four matrix equations:

$$
\begin{aligned}
A \sigma & =\sigma A \\
B N & =\sigma B \\
C \sigma & =N C \\
N D & =D N .
\end{aligned}
$$

We are going to show only that $B=0$ since for $C$ the idea of the proof is the same.

First of all, if $N=0$, then it immediately follows from the above equations that $B=0$, so we assume that $N$ is a nonzero nilpotent matrix. Let $r$ be the smallest integer such that $B N^{r}=0$. If $r=1$, then since $\sigma$ is invertible, it follows from equation (20) that $B=0$, so we continue with the assumption that $r>1$. In this case, by multiplying equation (20) with $N^{r-1}$ on the right we obtain $\sigma B N^{r-1}=B N^{r}=0$. By the same token, since $\sigma$ is invertible, we see that $B N^{r-1}=0$. But this contradicts with our assumption on the minimality of $r$. Therefore, $r=1$, hence $B=0$. Since both of $B$ and $C$ are 0 , the proof follows from equations (19) and (22).

By Proposition 3.1 and Remark 2.4, we see that if a partial transformation $f \in \mathcal{P}_{n}$ represents a loop-augmented forest, then its matrix form is as in (18). By this notation, as an application of Theorem 3.2, we will now record a representation theoretic generalization of the famous Knuth-Sagan hook length formula.

COROLLARY 3.3. Let $f$ be partial transformation representing a loop-augmented forest as in (18). If $\left(1^{m_{1}} 2^{m_{2}} \ldots k^{m_{k}}\right)$ is the conjugacy type of the permutation $\sigma$, then the dimension of $o(f)$, the $\mathcal{S}_{n}$ representation defined on the orbit of $f$ is equal to

$$
\frac{n !}{k !} \frac{\prod_{j} j^{m_{j}}\left(m_{j} !\right)}{\left(\prod_{a \in \tau} \prod_{b \in \gamma_{a}} m(a ; b) !\right)},
$$

where $m(a ; b)=m_{\tau}(a ; b)$ is as defined in $(6)$.

Proof. By Theorem 3.2 we know that the $\mathcal{S}_{n}$ representation defined by $f$ is isomorphic to $\mathbb{C}\left[\mathcal{S}_{n} / Z(\sigma) \times \operatorname{Stab}_{\mathcal{S}_{n-k}}(\tau)\right]$, hence its dimension is given by $\left.n ! /\left(|Z(\sigma)| \mid \operatorname{Stab}_{\mathcal{S}_{n-k}}(\tau)\right] \mid\right)$. We express this number in a different way:

$$
\begin{aligned}
\operatorname{dim}_{\mathbb{C}} \mathcal{S}_{n} \cdot f & =\frac{n !}{|Z(\sigma)|\left|\operatorname{Stab}_{\mathcal{S}_{n-k}}(\tau)\right|} \\
& =\left(\begin{array}{l}
n \\
k
\end{array}\right) \frac{k !}{|Z(\sigma)|} \frac{(n-k) !}{\left|\operatorname{Stab}_{\mathcal{S}_{n-k}}(\tau)\right|}
\end{aligned}
$$


Note that $|Z(\sigma)|$ is equal to $k ! /|C(\sigma)|$, where $C(\sigma)$ is the conjugacy class of $\sigma$ in $\mathcal{S}_{k}$. The cardinality of $C(\sigma)$ is equal to $k ! / \prod_{j} j^{m_{j}}\left(m_{j} !\right)$. Thus, the rest of the proof follows from (6) and (23).

\section{Characters}

Let $f$ be a partial transformation in $\mathcal{N}(n, k)$ and let $H(f)$ denote the stabilizer subgroup $\operatorname{Stab}_{\mathcal{S}_{n}}(f)$. As a consequence of Theorem 3.2 we see that the vector space $o(f)$ is $\mathcal{S}_{n}$-equivariantly isomorphic to the vector space $\mathbb{C}\left[\mathcal{S}_{n} / H(f)\right]$ and that $H(f) \simeq$ $Z(\sigma) \times \operatorname{Stab}_{\mathcal{S}_{n-k}}(\tau)$ for some permutation $\sigma \in \mathcal{S}_{k}$ and a nilpotent partial transformation $\tau \in \mathcal{C}_{n-k}$. The $\mathcal{S}_{n}$-module structure on $\mathbb{C}\left[\mathcal{S}_{n} / H(f)\right]$ is given by the left translation action of $\mathcal{S}_{n}$ on the cosets of $H(f)$. In other words, $o(f)$ is isomorphic to the induced trivial representation from $H(f)$ to $\mathcal{S}_{n}$. Since $H(f)$ decomposes as a direct product, the character of $o(f)$ is equal to the product of characters of the induced trivial representations on the corresponding ambient symmetric subgroups. We paraphrase the (isomorphism of) representations in induction notation:

$$
\operatorname{Ind}_{Z(\sigma) \times \operatorname{Stab}_{\mathcal{S}_{n-k}}(\tau)}^{\mathcal{S}_{n}} \mathbb{1} \simeq \operatorname{Ind}_{Z(\sigma)}^{\mathcal{S}_{k}} \mathbb{1} \otimes \operatorname{Ind}_{\operatorname{Stab}_{\mathcal{S}_{n-k}}(\tau)}^{\mathcal{S}_{n-k}} \mathbb{1}
$$

Notation. Let $H \subset G$ be a subgroup. We denote by $\chi_{H, G}$ the character of the induced trivial representation $\operatorname{Ind}_{H}^{G} \mathbb{1}$. If $\tau$ is a loop-augmented forest on $m$ vertices, then we denote the character of the induced trivial representation from $\operatorname{Stab}_{\mathcal{S}_{m}}(\tau)$ to $\mathcal{S}_{m}$ by $\chi_{o(\tau)}$.

LEMMA 4.1. We preserve our notation from the previous paragraph and let $f=\left(\begin{array}{cc}\sigma & 0 \\ 0 & \tau\end{array}\right)$ denote a partial function representing a loop-augmented forest. In this case the character of $o(f)$ has the following factorization:

$$
\chi_{o(f)}=\chi_{Z(\sigma), \mathcal{S}_{k}} \cdot \chi_{o(\tau)} .
$$

Proof. The proof is a straightforward consequence of (24).

The set of cosets of the subgroup $Z(\sigma)$ in $\mathcal{S}_{k}$ is in natural bijection with the set of conjugates of $\sigma \in \mathcal{S}_{k}$. In other words, viewed as an $\mathcal{S}_{n}$-set, $\mathcal{S}_{k} / Z(\sigma)$ is identified with the conjugacy class of $\sigma$ in $\mathcal{S}_{k}$. As we mentioned before, the conjugacy classes $\left(\right.$ of $\mathcal{S}_{k}$ ) are parametrized by the partitions (of $k$ ). Each conjugacy class in $\mathcal{S}_{k}$ is an $\mathcal{S}_{k}$-set, therefore, it defines a representation of $\mathcal{S}_{k}$. Moreover, such a representation is irreducible. By the Frobenius characteristic map the irreducible representation on a conjugacy class that is indexed by a partition $\nu$ of $k$ is mapped to the Schur function $s_{\nu}$. Clearly, this argument takes care of the character of the induced representation $\operatorname{Ind}_{Z(\sigma)}^{\mathcal{S}_{k}} \mathbb{1}$ in $(24)$. For the other tensor factor, we refer to our earlier work: [1, Corollary 6.5] states that if $\lambda=\left(\lambda_{1}, \ldots, \lambda_{r}\right)$ is a partition of $n-k$ and $\tau$ is a rooted forest consisting of $\lambda_{1}$ copies of a rooted tree $\tau_{1}, \lambda_{2}$ copies of a rooted tree $\tau_{2}$ and so on, then the character of $o(\tau)$ is given by

$$
\chi_{o(\tau)}=\left(\chi^{\left(\lambda_{1}\right)}\left[\chi_{o\left(\tau_{1}\right)}\right]\right) \cdot\left(\chi^{\left(\lambda_{2}\right)}\left[\chi_{o\left(\tau_{2}\right)}\right]\right) \cdots\left(\chi^{\left(\lambda_{r}\right)}\left[\chi_{o\left(\tau_{r}\right)}\right]\right) .
$$

We summarize these observations as follows:

THEOREM 4.2. Let $f$ be a partial function representing a loop-augmented forest on $n$ vertices. Then $f$ is similar to a block diagonal matrix of the form $\left(\begin{array}{cc}\sigma & 0 \\ 0 & \tau\end{array}\right)$ for some $\sigma \in \mathcal{S}_{k}$ and $\tau \in \mathcal{M}\left(n-k, \mathbf{0}_{n-k}\right)$. Furthermore, if $\nu$, which is a partition of $k$, is the conjugacy type of $\sigma$ in $\mathcal{S}_{k}$ and if the underlying rooted forest of the nilpotent partial transformation $\tau$ has $\lambda_{1}$ copies of the rooted tree $\tau_{1}, \lambda_{2}$ copies of the rooted forest $\tau_{2}$ and so on, then the character of $o(f)$ is given by

$$
\chi_{o(f)}=\chi^{\nu} \cdot \chi_{o(\tau)}=\chi^{\nu} \cdot\left(\chi^{\left(\lambda_{1}\right)}\left[\chi_{o\left(\tau_{1}\right)}\right]\right) \cdot\left(\chi^{\left(\lambda_{2}\right)}\left[\chi_{o\left(\tau_{2}\right)}\right]\right) \cdots\left(\chi^{\left(\lambda_{r}\right)}\left[\chi_{o\left(\tau_{r}\right)}\right]\right) .
$$


Proof. The proof follows from the discussion in the previous paragraph and Lemma 4.1.

\section{Nondiagonal Idempotents and Their Stabilizer Subgroups}

Let $e$ denote the $i$-th constant idempotent $c^{(i)}$. Observe that $e$ stays unchanged by multiplication by permutations on the right and therefore the conjugation action on $e$ is given by the left multiplication action; this is equivalent to the "standard representation" of $\mathcal{S}_{n}$ on $\mathbb{C}^{n}$. In particular, the stabilizer subgroup of $e$ is isomorphic to $\mathcal{S}_{1} \times \mathcal{S}_{n-1} \simeq \mathcal{S}_{n-1}$.

DEFINITION 5.1. Let e be an idempotent with $k 1$ 's, for $0<k \leqslant n$, on its $i$-th row and 0 's elsewhere. Then there exists $\sigma \in \mathcal{S}_{n}$ such that the nonzero entries of $\sigma e \sigma^{-1}$ are in the first row and in the first $k$ columns. In this case, we call $\sigma e \sigma^{-1}$ the standard form of $e$.

Note that a constant idempotent $c^{(r)}$ is already in its standard form. Note also that the standard form of an idempotent is unique. We will show in an algorithmic way that it is always possible to determine the permutation $\sigma$ that leads to the standard form of $e$.

ExAmple 5.2. Let $e$ denote the idempotent $\left(\begin{array}{lll}0 & 0 & 0 \\ 0 & 0 & 0 \\ 1 & 0 & 1\end{array}\right)$. Then the standard form of $e$ is given by $\sigma e \sigma^{-1}=\left(\begin{array}{lll}1 & 1 & 0 \\ 0 & 0 & 0 \\ 0 & 0 & 0\end{array}\right)$, where $\sigma$ is the permutation $(2,3)(1,3)$.

We proceed to explain our algorithmic construction of the permutation $\sigma$ for the idempotent $e$ as in Definition 5.1.

Step 1. If $i=1$, then start with Step 2. Assuming that $i>1$, since $e$ is an idempotent, we see that the $i$-th entry in the $i$-th row of $e$ is 1 . We apply the transposition $\sigma_{1}:=(1, i)$ to $e$ to bring its nonzero entry at the $(i, i)$-th position to the $(1,1)$-th position. In fact, the application of $\sigma_{1}$ to $e$ interchanges the $i$-th row with the first row and it interchanges the $i$-th column with the first column. We denote the resulting idempotent by $e_{1}$ and we move Step 2 .

Step 2. We assume that $i=1$ and we look for the first occurrence of 1 in the first row (of course, we skip the one in the first position). If this 1 is at the $j$-th position with $j>2$, then we apply the transposition $\sigma_{2}:=(2, j)$ to $e_{1} . \sigma_{2}$ interchanges the second and the $j$-th rows and it interchanges the second and the $j$-th columns in $e_{1}$. Thus, the 2-nd entry of the first row of $e_{2}:=\sigma_{2} \cdot e_{1}$ is now 1 . We repeat this process, by choosing transpositions, $\sigma_{3}, \sigma_{4}, \ldots$ until we move all of the 1 's in the first row to the left of the row. In summary, by conjugating $e$ by a product $\sigma_{s} \cdots \sigma_{1}$ of not all disjoint transpositions, we bring $e$ to its standard form, the idempotent having 1's in the first $k$ entries of its first row.

LEMMA 5.3. Let $e$ be an idempotent from $\mathcal{P}_{n}$ and let $j_{1} \leqslant \cdots \leqslant j_{r}$ denote the indices of those rows of e which have nonzero entries in them. If $\alpha_{i}(i=1, \ldots, r)$ denotes the number of 1's in the $j_{i}$-th row, then there exists a permutation $\sigma \in \mathcal{S}_{n}$ such that $\sigma e \sigma^{-1}$ is a block diagonal matrix of the form

$$
e=c^{\left(\alpha_{1}\right)} \oplus \cdots \oplus c^{\left(\alpha_{r}\right)} \oplus \mathbf{0}_{m}
$$

where $\mathbf{0}_{m}$ is the square 0 matrix of dimension $m:=n-\sum \alpha_{i}$.

Proof. The proof uses induction by first applying the standardization procedure outlined in the above paragraphs to the row $j_{1}$. Instead of repeating what is written 
above, we demonstrate this procedure by an example.

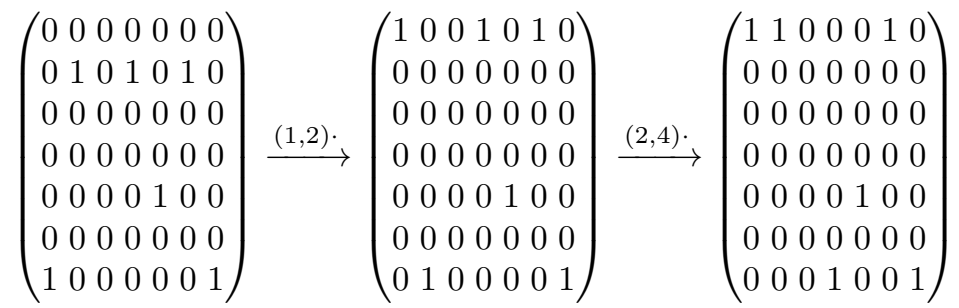

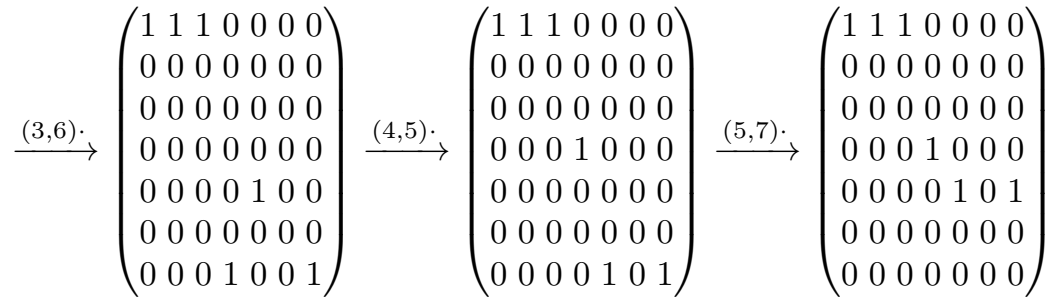

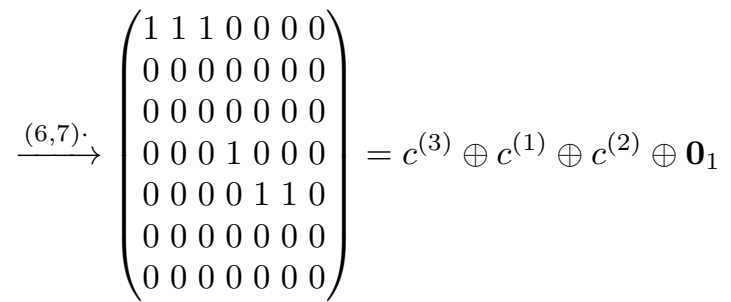

The proof of our next observation is now straightforward; it follows from the similar arguments as above.

Corollary 5.4. Let e be an idempotent from $\mathcal{P}_{n}$. Then there exists a permutation $\sigma \in \mathcal{S}_{n}$ such that $\sigma e \sigma^{-1}$ is a block diagonal matrix of the form

$$
\sigma e \sigma^{-1}=c^{\left(\beta_{1}\right)} \oplus \cdots \oplus c^{\left(\beta_{r}\right)} \oplus \mathbf{0}_{m},
$$

where $\mathbf{0}_{m}$ is the square 0 matrix of dimension $m:=n-\sum \beta_{i}$ and $\beta_{1} \geqslant \beta_{2} \cdots \geqslant \beta_{r}$.

We now generalize our previous definition of the "standard form of an idempotent". For an arbitrary idempotent $e \in \mathcal{P}_{n}$, we call its conjugate $\sigma e \sigma^{-1}=c^{\left(\beta_{1}\right)} \oplus \cdots \oplus c^{\left(\beta_{r}\right)} \oplus$ $\mathbf{0}_{m}$ as in (26) the standard form of $e$ and denote it by $s t(e)$. An idempotent $e$ is called standard if it satisfies the equation $x=s t(x)$. Our next task is to compute the stabilizer of a standard idempotent.

THEOREM 5.5. Let $\beta_{1} \geqslant \beta_{2} \geqslant \cdots \geqslant \beta_{r}$ be a sequence of positive integers and let $e \in \mathcal{P}_{n}$ be a standard idempotent in which $c^{\left(\beta_{1}\right)}$ appears $m_{1}$ times, $c^{\left(\beta_{2}\right)}$ appears $m_{2}$ times, and so on. If $\sum_{i=1}^{s} m_{i} \beta_{i}=n$, then the stabilizer subgroup in $\mathcal{S}_{n}$ of $e$ is

$$
\operatorname{Stab}_{\mathcal{S}_{n}}(e)=\left(\mathcal{S}_{m_{1}} \text { 々 } \mathcal{S}_{\beta_{1}-1}\right) \times\left(\mathcal{S}_{m_{2}} \text { 々 } \mathcal{S}_{\beta_{2}-1}\right) \times \cdots \times\left(\mathcal{S}_{m_{s}} \text { 々 } \mathcal{S}_{\beta_{s}-1}\right) \text {. }
$$

Proof. We start with the simple case that $e=c^{\left(\beta_{1}\right)} \oplus \cdots \oplus c^{\left(\beta_{1}\right)} \oplus c^{\left(\beta_{2}\right)} \oplus \cdots \oplus c^{\left(\beta_{2}\right)}$, where $c^{\left(\beta_{1}\right)}$ appears $m_{1}$ times and $c^{\left(\beta_{2}\right)}$ appears $m_{2}$ times. If $\sigma \in \mathcal{S}_{n}$ stabilizes $e$, then we have $\sigma e=e \sigma$. We decompose $\sigma$ into 4 blocks

$$
\sigma=\left(\begin{array}{ll}
\sigma_{11} & \sigma_{12} \\
\sigma_{21} & \sigma_{22}
\end{array}\right)
$$

where $\sigma_{11}$ is of dimension $m_{1} \beta_{1}$ and $\sigma_{22}$ is of dimension $m_{2} \beta_{2}$. For simplicity of notation, let us denote $c^{\left(\beta_{1}\right)} \oplus \cdots \oplus c^{\left(\beta_{1}\right)}$ by $c_{11}$ and denote $c^{\left(\beta_{2}\right)} \oplus \cdots \oplus c^{\left(\beta_{2}\right)}$ by $c_{22}$. By a simple block-matrix multiplication we observe that

(1) $\sigma_{11}$ commutes with $c_{11}$; 
(2) $\sigma_{22}$ commutes with $c_{22}$;

(3) $\sigma_{12} c_{22}=c_{11} \sigma_{12}$ which implies that $\sigma_{12}$ is a 0 -matrix since $c^{\left(\beta_{1}\right)}$ and $c^{\left(\beta_{2}\right)}$ are of different dimensions;

(4) $\sigma_{21} c_{11}=c_{22} \sigma_{21}$ which implies that $\sigma_{21}$ is a 0 -matrix since $c^{\left(\beta_{1}\right)}$ and $c^{\left(\beta_{2}\right)}$ are of different dimensions.

The last two items shows that $\operatorname{Stab}_{\mathcal{S}_{n}}(e)=\operatorname{Stab}_{\mathcal{S}_{m_{1} \beta_{1}}}\left(c_{11}\right) \oplus \operatorname{Stab}_{\mathcal{S}_{m_{2} \beta_{2}}}\left(c_{22}\right)$. Thus, $\sigma_{i i}$ belongs to $\mathcal{S}_{m_{i} \beta_{i}}$ for $i=1,2$.

To show that $\operatorname{Stab}_{\mathcal{S}_{m_{i} \beta_{i}}}\left(c_{i i}\right) \simeq \mathcal{S}_{m_{i}}<\mathcal{S}_{\beta_{i}-1}(i=1,2)$, we assume without loss of generality that $i=1$ and we decompose $\sigma_{11}$ into $m_{1}^{2}$ square blocks as in

$$
\sigma_{11}=\left(\begin{array}{ccc}
\tau_{11} & \cdots & \tau_{1 m_{1}} \\
\vdots & \ddots & \vdots \\
\tau_{m_{1} 1} & \cdots & \tau_{m_{1} m_{1}}
\end{array}\right) .
$$

Note that each $\tau_{i j}$ is a rook matrix. Since $c_{11}$ has copies of $c^{\left(\beta_{1}\right)}$ 's along its main diagonal and zero blocks elsewhere, it follows from the commutation relation $\sigma_{11} c_{11}=$ $c_{11} \sigma_{11}$ that

$$
\tau_{i j} c^{\left(\beta_{1}\right)}=c^{\left(\beta_{1}\right)} \tau_{i j}
$$

for all $i, j=1, \ldots, m_{1}$. It is not difficult to verify that unless $\tau_{i j}$ is a permutation matrix with 1 at its $(1,1)$-th entry, in order for relation (28) to hold, $\tau_{i j}$ must be a zero matrix. In other words, in each row (of blocks) in (27) there exists exactly 1 block which is a permutation matrix of dimension $\beta_{1}$ with 1 at its $(1,1)$-th entry. It is not difficult to verify that a permutation matrix $\tau \in \mathcal{S}_{m_{1} \beta_{1}}$ with $m_{1}^{2}$ square blocks just as described satisfies the commutation relation $\tau c_{11}=c_{11} \tau$, also. But such a permutation $\tau$ is uniquely determined by a permutation $\tau^{\prime} \in \mathcal{S}_{m_{1}}$ describing the positions of nonzero blocks of $\tau$ and in addition $m_{1}^{2}$ permutation matrices $\tau_{i j}^{\prime} \in \mathcal{S}_{\beta_{1}-1}$ which forms that lower-right sub-matrices of the nonzero blocks. This is precisely our definition of $\mathcal{S}_{m_{1}} \prec \mathcal{S}_{\beta_{1}-1}$.

Corollary 5.6. If $e \in \mathcal{P}_{n}$ is an idempotent, then there exists a multiset of integers $\left\{\beta_{1}^{m_{1}}, \ldots, \beta_{s}^{m_{s}}\right\}$ and $m \in \mathbb{N}$ such that

(1) $m+\sum_{i=1}^{s} m_{i} \beta_{i}=n$;

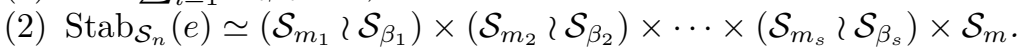

Proof. We know from Corollary 5.4 that by conjugating with an appropriate permutation $\sigma \in \mathcal{S}_{n}$ we get $\sigma e \sigma^{-1}=\widetilde{e} \oplus \mathbf{0}_{m}$, where $\widetilde{e}$ is a standard idempotent in $\mathcal{P}_{k}$ for some $1 \leqslant k \leqslant n$ and $m=n-k$. The rest of the proof follows from Theorem 5.5.

\section{The Conjecture}

Throughout this section, when the parts of a partition $\lambda=\left(\lambda_{1}, \ldots, \lambda_{k}\right)$ satisfy $\lambda_{1}=$ $\cdots=\lambda_{k}$, we will write $\lambda=\lambda_{1}^{k}$.

Let $\tau$ be a rooted forest with the Frobenius character $F_{\tau}$. Recall from Introduction that adding a root to $\tau$ has the effect of multiplying $F_{\tau}$ with $s_{1}$. Recall also that, if $\tau$ is a rooted tree, then the Frobenius character of the rooted forest that is comprised of $m$ copies of $\tau$ is equal to $s_{m}\left[F_{\tau}\right]$.

EXAMPLE 6.1. Since $s_{1}$ is equal to the power-sum symmetric function $p_{1}$, the Frobenius character of $n-1$ disjoint nodes (for $n \geqslant 2$ ) is $s_{n-1}\left[s_{1}\right]=s_{n-1}$.

EXAMPLE 6.2. The Frobenius character of the rooted tree that is obtained from $n-1$ disjoint nodes (for $n \geqslant 2$ ) by attaching them to a root is $s_{1} s_{n-1}$. 
EXAMPLE 6.3. The Frobenius character of the rooted forest that is obtained by taking $m$ copies of the rooted tree of Example 6.2 is $s_{m}\left[s_{1} s_{n-1}\right]$.

The rest of our paper is devoted to a proof of our conjectured extension of Foulkes's conjecture in the special case where $m=2$. In the light of Examples 6.1-6.3, our general conjecture can be stated in the language of symmetric functions as follows.

CONJECTURE 6.4. If $n>m \geqslant 2$, then for any $\lambda$ which has more than two parts,

$$
\left\langle s_{n}\left[s_{1} s_{m-1}\right], s_{\lambda}\right\rangle \geqslant\left\langle s_{m}\left[s_{1} s_{n-1}\right], s_{\lambda}\right\rangle .
$$

We will prove this conjecture in the case where $m=2$. In particular, we shall show that for all $n>2$, and $\lambda \neq(2,2 n-2)$,

$$
\left\langle s_{n}\left[s_{1} s_{1}\right], s_{\lambda}\right\rangle \geqslant\left\langle s_{2}\left[s_{1} s_{n-1}\right], s_{\lambda}\right\rangle \text {. }
$$

and

$$
\left\langle s_{n}\left[s_{1} s_{1}\right], s_{(2,2 n-2)}\right\rangle=2 \text {, and }\left\langle s_{2}\left[s_{1} s_{n-1}\right], s_{(2,2 n-2)}\right\rangle=3 .
$$

Before we can carry out our computations, we need to recall Littlewood's formulas for the plethysms $s_{n}\left[s_{2}\right]$ and $s_{n}\left[e_{2}\right]$, the Murnaghan-Nakayama rule to compute the product of power symmetric function $p_{n}$ times a Schur function $s_{\mu}$, and Chen's rule [2] which allows us to compute the plethysm $p_{k}\left[s_{n}\right]$.

We say that a partition $\lambda=\left(\lambda_{1}, \ldots, \lambda_{k}\right)$ is even if $\lambda_{i}$ is even for all $i$. Then Littlewood [5] proved the following two results.

$$
s_{n}\left[s_{2}\right]=\sum_{\substack{\lambda \vdash 2 n \\ \lambda \text { even }}} s_{\lambda}
$$

and

$$
s_{n}\left[e_{2}\right]=\sum_{\substack{\lambda \vdash 2 n \\ \lambda^{\prime} \text { even }}} s_{\lambda}
$$

Next we introduce the notion of rim hook tableaux, special rim hook tabloids, and transposed special rim hook tabloids.

Given a Ferrers diagram $F(\lambda)$ of a partition, a rim hook $h$ of $\lambda$ is a consecutive sequence of cells along the northeast boundary of $\lambda$ such that any two consecutive cells of $h$ share an edge and the removal of the cells of $h$ from $\lambda$ results in a Ferrers diagram of another partition. We let $r(h)$ denote the number of rows of $h$ and $c(h)$ denote the number of columns of $h$. We say that $h$ is special if $h$ has at least one cell in the first column of $F(\lambda)$ and $h$ is transposed special if $h$ has at least one cell in the first row of $F(\lambda)$. We define the sign of $h, \operatorname{sgn}(h)$, to be

$$
\operatorname{sgn}(h)=(-1)^{r(h)-1} \text {. }
$$

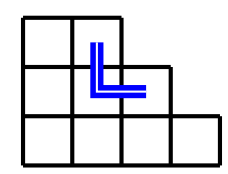

Figure 6. A rim hook of $(2,3,4)$.

For example, Figure 6 gives a rim hook $h$ of shape $(2,3,4)$ which is neither special nor transposed special. Since $r(h)=2$, then $\operatorname{sgn}(h)=(-1)^{2-1}=-1$. Figure 7 (a) pictures all the special rim hooks of $\lambda=(2,2,4)$ and Figure $7(\mathrm{~b})$ pictures all the transposed special rim hooks of $\lambda=(2,2,4)$. 
(a)
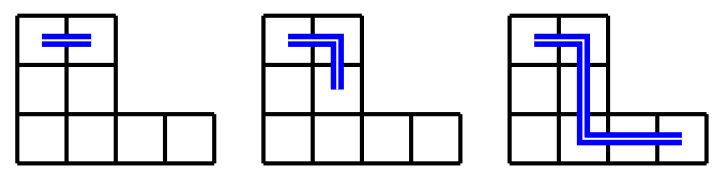

(b)
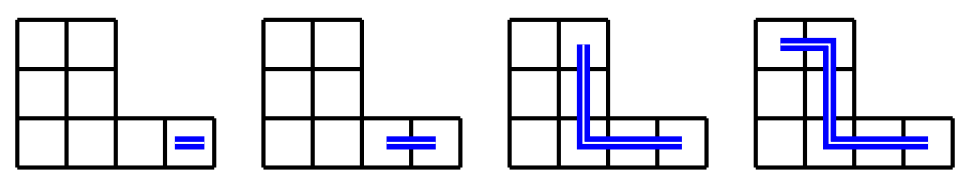

FigURE 7. The special and transposed special rim hooks of $(2,2,4)$.

The Murnaghan-Nakayama rule gives us a rule for the Schur function expansion of a power symmetric function $p_{n}$ times a Schur function $s_{\mu}$. That is,

$$
p_{n} s_{\mu}=\sum_{\substack{\mu \subseteq \lambda \\ \lambda / \mu \text { is a rim hook of } \lambda \text { of size } n}} \operatorname{sgn}(\lambda / \mu) s_{\lambda} .
$$

A rim hook tabloid of shape $\lambda$ and type $\mu=\left(\mu_{1}, \ldots, \mu_{k}\right)$ is a filling of the Ferrers diagram of $\lambda$ with rim hooks $\left(s_{1}, \ldots, s_{k}\right)$ such that $\left(\left|s_{1}\right|, \ldots,\left|s_{k}\right|\right)$ is a rearrangement of $\left(\mu_{1}, \ldots, \mu_{k}\right)$ where $\left|s_{i}\right|$ denotes the number of cells of $s_{i}$. To be more precise, one can think of a rim hook tabloid $T$ as a sequences of shapes $\left\{\varnothing=\lambda^{(0)} \subset\right.$ $\left.\lambda^{(1)} \subset \cdots \subset \lambda^{(k)}=\lambda\right\}$ such that for all $i \geqslant 1, \lambda^{(i)} / \lambda^{(i-1)}$ is a rim hook of $\lambda^{(i)}$ and $\left(\left|\lambda^{(1)} / \lambda^{(0)}\right|,\left|\lambda^{(2)} / \lambda^{(1)}\right|, \ldots,\left|\lambda^{(k)} / \lambda^{(k-1)}\right|\right)$ is a rearrangement of $\left(\mu_{1}, \ldots, \mu_{k}\right) . T$ is called a special (t-special) rim hook tabloid if for all $i \geqslant 1, \lambda^{(i)} / \lambda^{(i-1)}$ is a special (transposed special) rim hook. The sign of $T, \operatorname{sgn}(T)$ is defined to be

$$
\operatorname{sgn}(T)=\prod_{i=1}^{k} \operatorname{sgn}\left(\lambda^{(i)} / \lambda^{(i-1)}\right) .
$$

We emphasize however that the rim hook tabloid $T$ of shape $\lambda$ is the filling of $F(\lambda)$ and is not the sequence of shapes $\left\{\varnothing=\lambda^{(0)} \subset \lambda^{(1)} \subset \cdots \subset \lambda^{(k)}=\lambda\right\}$. That is, in Figure 8 pictures a rim hook tabloid $T$ of shape $\lambda=(2,2,4)$ and type $(2,3,3)$ whose sign is $(-1)^{2-1}(-1)^{2-1}(-1)^{1-1}=1$. There are two sequences of shapes that can be associated to $T$, namely,

$$
\begin{aligned}
T & =\{\varnothing \subset(1,2) \subset(2,2,2) \subset(2,2,4)\} \\
\text { and } \quad T & =\{\varnothing \subset(1,2) \subset(1,4) \subset(2,2,4)\}
\end{aligned}
$$

depending on whether one takes to top rim hook to be the second or third rim hook. Of course, if $T$ is a special rim hook tabloid or a $t$-special rim hook tabloid, then there is a unique sequence of shapes $\left\{\varnothing=\lambda^{(0)} \subset \lambda^{(1)} \subset \cdots \subset \lambda^{(k)}=\lambda\right\}$ that can be associated to $T$.

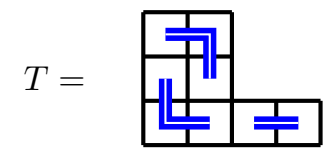

Figure 8. A rim hook tabloid with associated sequences of shapes. 
Chen's rule [2] states that $(33)$

$$
\left\langle p_{k}\left[s_{n}\right], s_{\lambda}\right\rangle= \begin{cases}\operatorname{sgn}(T) & \text { if } T \text { is a special rim hook tabloid of shape } \lambda \text { and type } k^{n} \\ 0 & \text { otherwise }\end{cases}
$$

Thus to compute $p_{k}\left[s_{n}\right]=s_{n}\left[p_{k}\right]$, we need only generate all the $t$-special rim hook tabloids $T$ of type $k^{n}$ and replace $T$ by $\operatorname{sgn}(T) s_{s h(T)}$ where $\operatorname{sh}(T)$ denotes the shape of $T$. For example, Figure 9 pictures all the $t$-special rim hook tabloids of type $3^{2}$ where instead of drawing a Ferrers diagram, we have indicated the cells of the Ferrers diagram by dots. Thus $p_{3}\left[s_{2}\right]=s_{2}\left[p_{3}\right]=s_{\left(2^{3}\right)}-s_{(1,2,3)}+s_{\left(1^{2}, 4\right)}+s_{(3,3)}-s_{(1,5)}+s_{(6)}$.
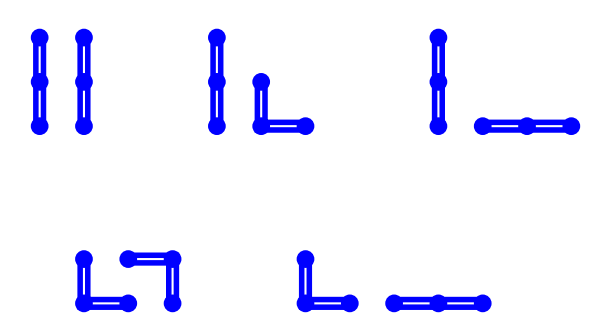

FigurE 9. The $t$-special rim hook tabloids of type $\left(3^{2}\right)$.

The computation of $p_{2}\left[s_{n}\right]$ is particularly straightforward via Chen's rule. That is, a $t$-special rim hook tabloid of type $2^{n}$ consists of bunch of vertical rim hooks followed by a bunch of horizontal rim hooks as pictured in Figure 10.

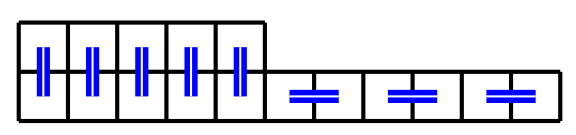

FiguRE 10. $t$-special rim hook tabloids of type $2^{n}$.

It easily follows that

$$
p_{2}\left[s_{n}\right]=\sum_{0 \leqslant a \leqslant n}(-1)^{a} s_{(a, 2 n-a)} .
$$

We want to compare $s_{2}\left[s_{1} s_{n-1}\right]$ to $s_{n}\left[s_{1} s_{1}\right]$. Obviously, the two expressions are equal when $n=2$. Our first goal is to prove the following theorem which gives an explicit formula for the Schur function expansion of $s_{2}\left[s_{1} s_{n-1}\right]$.

Notation. For any logical statement $A$, we set

$$
\chi(A)= \begin{cases}1 & \text { if } A \text { is true } \\ 0 & \text { if } A \text { is false }\end{cases}
$$


THEOREM 6.5. For $n \geqslant 3$,

$$
\begin{aligned}
s_{2}\left[s_{1} s_{n-1}\right]=\sum_{\substack{1 \leqslant a \leqslant n-1 \\
a \text { odd }}} s_{\left(1^{2}, a, 2 n-2-a\right)}+\sum_{\substack{2 \leqslant a \leqslant n-1 \\
a \text { even }}} s_{(2, a, 2 n-2-a)} \\
+s_{\left(1^{2}, 2 n-2\right)}+\sum_{2 \leqslant a \leqslant n-1} 2 s_{(1, a, 2 n-1-a)} \\
+s_{(1,2 n-1)}+\sum_{\substack{2 \leqslant a \leqslant n-1 \\
a \text { even }}} 3 s_{(a, 2 n-a)}+\sum_{\substack{2 \leqslant a \leqslant n-1 \\
a \text { odd }}} s_{(a, 2 n-a)} \\
+2 \chi(n \text { even }) s_{(n, n)}+s_{(2 n)} .
\end{aligned}
$$

Proof. Note that by (34) and the Pieri rules we have

$$
\begin{aligned}
s_{2}\left[s_{1} s_{n-1}\right] & =\left(\frac{1}{2}\left(p_{1}^{2}+p_{2}\right)\right)\left[s_{1} s_{n-1}\right] \\
& =\frac{1}{2} s_{1}^{2}\left[s_{1} s_{n-1}\right]+\frac{1}{2} p_{2}\left[s_{1} s_{n-1}\right] \\
& =\frac{1}{2} s_{1}^{2} s_{n-1}^{2}+\frac{1}{2} p_{2} p_{2}\left[s_{n-1}\right] \\
& =\frac{1}{2}\left(s_{1}^{2}\left(\sum_{0 \leqslant a \leqslant n-1} s_{(a, 2 n-2-a)}\right)+p_{2}\left(\sum_{0 \leqslant a \leqslant n-1}(-1)^{a} s_{(a, 2 n-2-a)}\right)\right) .
\end{aligned}
$$

It is easy to see from the Pieri rules and the Murnaghan-Nakayama rules that the only shapes $\lambda$ of Schur functions $s_{\lambda}$ that can arise in the Schur function expansions of products of the from $s_{1}^{2} s_{(a, 2 n-2-a)}$ and $p_{2} s_{(a, 2 n-2-a)}$ are $\left(1^{2}, a, b\right),(2, a, b),(1, a, b)$, $(a, b)$, or $(2 n)$. We will consider each type of shape in turn. It follows from the Pieri rules that a product $s_{1}^{2} s_{(a, 2 n-2-a)}$ is the sum over all $s_{\lambda}$ where we first add single cell on the outside of $(a, 2 n-2-a)$ to get a shape $\mu$, which we will indicate by a cell containing the number 1 , and then adding a single cell on the outside of the $\mu$, which we will indicate by cell containing 2 .

By the Murnaghan-Nakayama rule, a product of the form $p_{2} s_{(a, 2 n-2-a)}$ is the sum over all $\pm s_{\lambda}$ that arises by putting a rim hook of length 2 along the outside of $(a, 2 n-2-a)$, which we will indicate by a rim hook colored red (and drawn as a single solid line), where the sign is plus if the rim hook is horizontal and -1 if the rim hook is vertical.

Case 1. $\lambda=\left(1^{2}, a, b\right)$ where $1 \leqslant a \leqslant n-1$. In this case, there is only one way to get a term of the form $s_{\left(1^{2}, a, 2 n-2-a\right)}$, where $1 \leqslant a \leqslant n-1$, from the products $s_{1}^{2} s_{n-1}^{2}$ and $p_{2}\left[s_{n-1}\right] p_{2}$ which are pictured in Figure 11. That is, it is easy to see that $s_{\left(1^{2}, a, 2 n-2-a\right)}$ can only arise in one way from $s_{1}^{2} s_{n-1}^{2}$ which comes the product $s_{1}^{2} s_{(a, 2 n-2-a)}$ by placing the cells with 1 and 2 as pictured at the top of Figure 11. Similarly, $s_{\left(1^{2}, a, 2 n-2-a\right)}$ can only arise in one way from which comes the product $p_{2}(-1)^{a} s_{(a, 2 n-2-a)}$ by placing the rim hook as pictured in red at the bottom of Figure 11. Hence we get a contribution of $(-1)^{a+1} s_{\left(1^{2}, a, 2 n-2-a\right)}$ from $p_{2} p_{2}\left[s_{n-1}\right]$. These two cancel out if $1 \leqslant a \leqslant n-1$ and $a$ is even and they give a contribution of $\frac{1}{2} 2 s_{\left(1^{2}, a, 2 n-2-a\right)}=s_{\left(1^{2}, a, 2 n-2-a\right)}$ if $a$ is odd.

Thus the Schur functions in Case 1 contribute

$$
\sum_{\substack{1 \leqslant a \leqslant n-1 \\ a \text { odd }}} s_{\left(1^{2}, a, 2 n-2-a\right)}
$$

to $s_{2}\left[s_{1} s_{n-1}\right]$. 


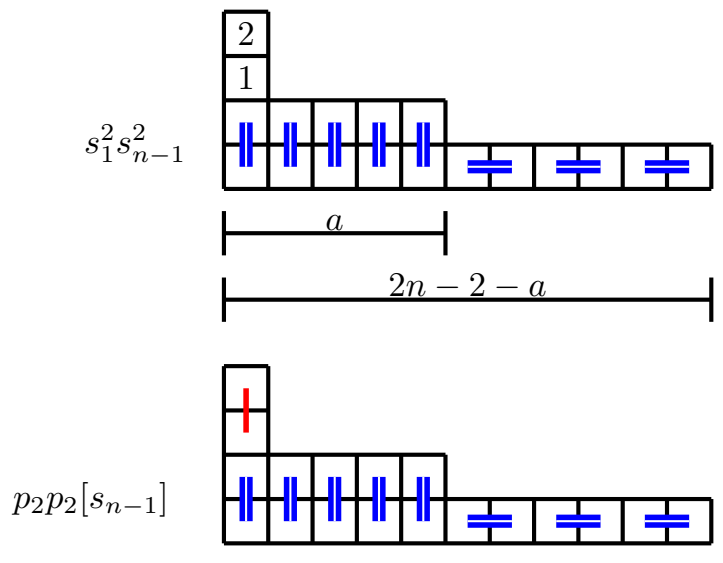

Figure 11. The diagrams for Case 1.

Case 2. $\lambda=(2, a, b)$ where $2 \leqslant a \leqslant n-1$. In this case, there is only one way to get a term of the form $s_{\left(1^{2}, a, 2 n-2-a\right)}$, where $1 \leqslant a \leqslant n-1$, from the products $s_{1}^{2} s_{n-1}^{2}$ and $p_{2}\left[s_{n-1}\right] p_{2}$ which are pictured in Figure 12 . That is, it is easy to see that $s_{\left(1^{2}, a, 2 n-2-a\right)}$ can only arise in one way from $s_{1}^{2} s_{n-1}^{2}$ which comes the product $s_{1}^{2} s_{(a, 2 n-2-a)}$ by placing the cells with 1 and 2 as pictured at the top of Figure 12. Similarly, $s_{\left(1^{2}, a, 2 n-2-a\right)}$ can only arise in one way from which comes the product $p_{2}(-1)^{a} s_{(a, 2 n-2-a)}$ by placing the rim hook as pictured in red at the bottom of Figure 12. Hence we get a contribution of $(-1)^{a} s_{\left(1^{2}, a, 2 n-2-a\right)}$ from $p_{2} p_{2}\left[s_{n-1}\right]$. This two cancel out if $1 \leqslant a \leqslant n-1$ and $a$ is odd and a contribution of $\frac{1}{2} 2 s_{\left(1^{2}, a, 2 n-2-a\right)}=s_{\left(1^{2}, a, 2 n-2-a\right)}$ if $a$ is even.

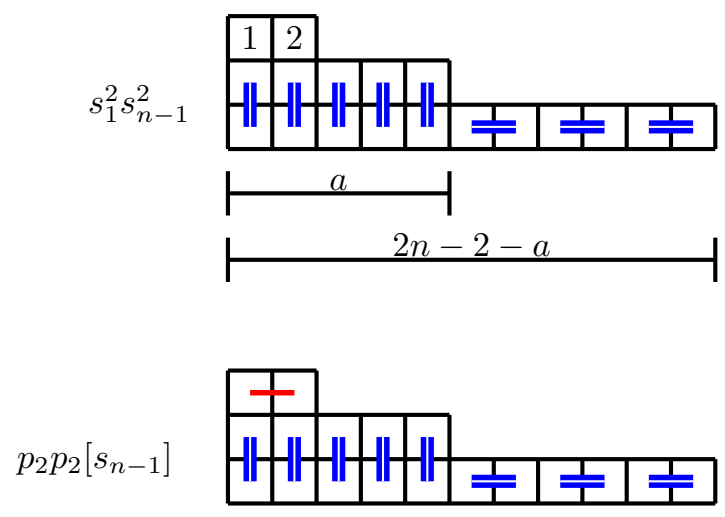

Figure 12. The diagrams for Case 2.

Thus the Schur functions in Case 2 contribute

to $s_{2}\left[s_{1} s_{n-1}\right]$.

$$
\sum_{\substack{2 \leqslant a \leqslant n-1 \\ a \text { even }}} s_{(2, a, 2 n-2-a)}
$$

Case 3. $\lambda=(1, a, 2 n-a-1)$ where $1 \leqslant a \leqslant n-1$. In this case, we must consider two subcases.

Subcase 3.1. $a=1$. Then $s_{\left(1^{2}, 2 n-2-a\right)}$ can arise in exactly three ways from $s_{1}^{2} s_{n-1}^{2}$, once from $s_{1}^{2} s_{2 n-2}$ and two ways from $s_{1}^{2} s_{(1,2 n-3)}$. These are pictured at the top of 
Figure 13. $s_{\left(1^{2}, 2 n-2-a\right)}$ can only arise in one way from $p_{2} p_{2}\left[s_{n-1}\right]$ which from the product $p_{2} s_{2 n-2}$ and comes with a sign of -1 . This possibility is pictured at the bottom Figure 13. Thus $s_{\left(1^{2}, 2 n-2\right)}$ appears once in the expansion of $s_{2}\left[s_{1} s_{n-1}\right]$.

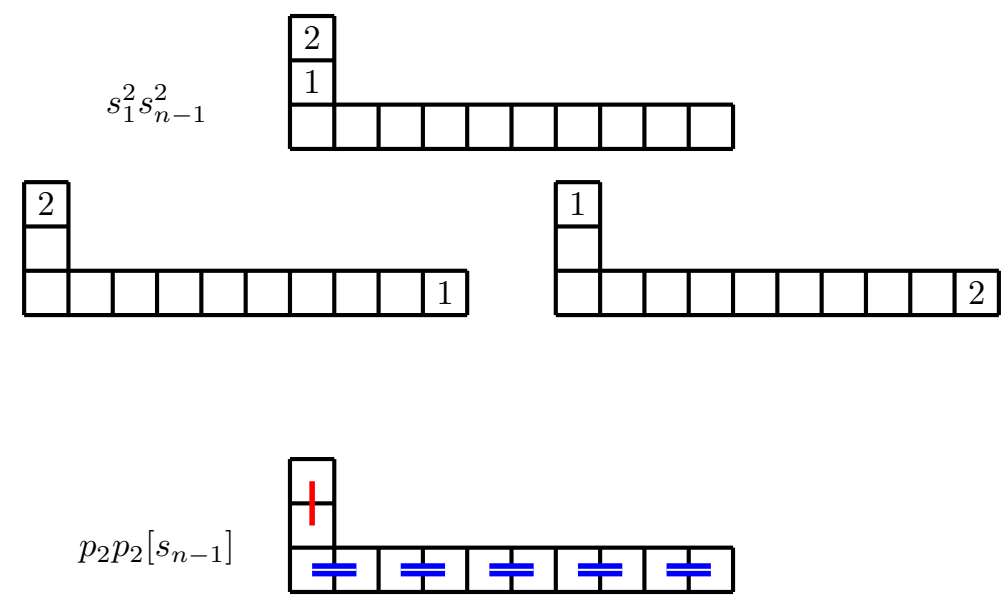

Figure 13. The diagrams for Case 3.1.

Subcase 3.2. $2 \leqslant a \leqslant n-1$. Then it is easy to see that we can not obtain a Schur function of shape $(1, a, 2 n-1-a)$ by adding a rim hook of size two on the outside of two row shape. Thus $s_{(1, a, 2 n-1-a)}$ does not appear in the expansion of $p_{2} p_{2}\left[s_{n-1}\right]$. However it can appear in the expansion of $s_{1}^{2} s_{n-1}$ in 4 different ways, namely, twice from the product $s_{1}^{2} s_{(a-1,2 n-a-1)}$ and twice from the product $s_{1}^{2} s_{(a, 2 n-a-2)}$. These are pictured in Figure 14. Thus in Subcase 3.2, we get an additional contribution of $2 \sum_{2 \leqslant a \leqslant n-1} s_{(1, a, 2 n-1-a)}$.

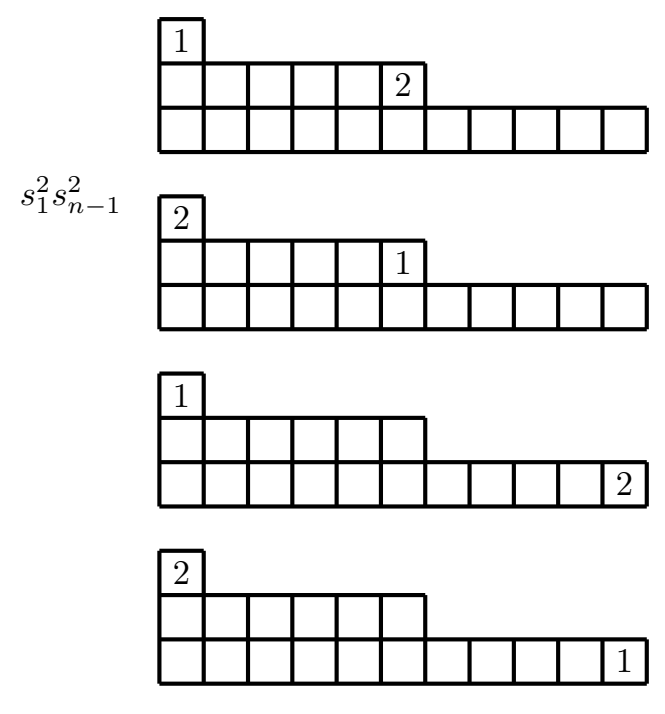

Figure 14. The diagrams for Case 3.2.

Thus the Schur functions in Case 3 contribute

$$
s_{\left(1^{2}, 2 n-2\right)}+2 \sum_{2 \leqslant a \leqslant n-1} s_{(1, a, 2 n-1-a)}
$$


to $s_{2}\left[s_{1} s_{n-1}\right]$.

Case 4. $\lambda=(a, 2 n-a)$ where $1 \leqslant a \leqslant n$. In this case, we have three subcases.

Subcase 4.1. $a=1$. In this case we have three ways to obtain $s_{(1,2 n-1)}$ from $s_{1}^{2} s_{n-1}^{2}$, namely, two ways from the product $s_{1}^{2} s_{(2 n-1)}$ and one way from the product $s_{1}^{2} s_{(1,2 n-3)}$. These three possibilities are pictured at the top of Figure 15 . There is only one way to obtain $s_{(1,2 n-1)}$ from $p_{2} p_{2}\left[s_{n-1}\right]$, namely, from the product $p_{2} s_{(1,2 n-3)}$ which comes with a sign of -1 . Thus Subcase 4.1 contributes a factor of $s_{(1,2 n-1)}$.
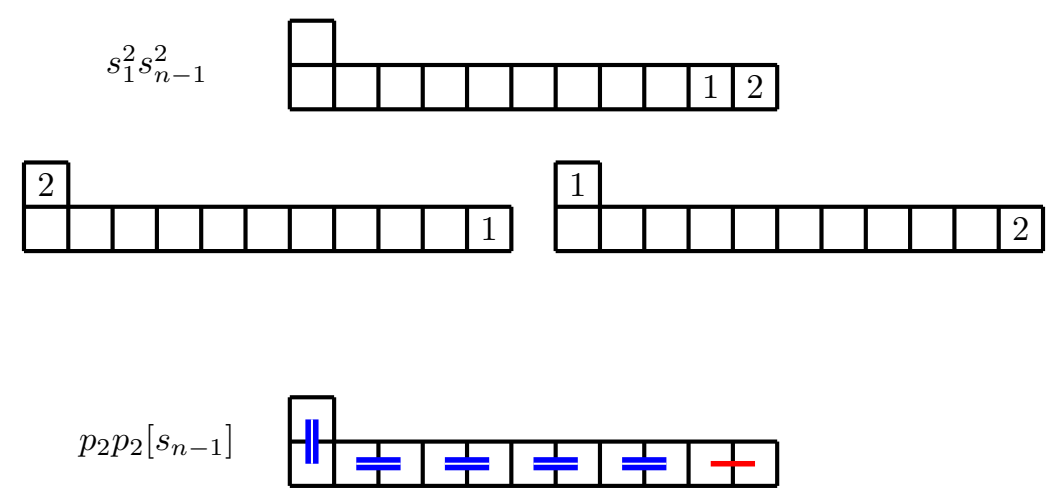

Figure 15. The diagrams for Case 4.1.

Subcase 4.2. $a=n$. In this case we have two ways to obtain $s_{(n, n)}$ from $s_{1}^{2} s_{n-1}^{2}$, namely, one way from the product $s_{1}^{2} s_{(n-2, n)}$ and one way from the product $s_{1}^{2} s_{(n-1, n-1)}$. These possibilities are pictured at the top of Figure 16. There are two ways to obtain $s_{(n, n)}$ from $p_{2} p_{2}\left[s_{n-1}\right]$, namely, once from the product $p_{2} s_{(n-2, n)}$ which comes with a sign of $(-1)^{n-2}$ and once from the product $p_{2} s_{(n-1, n-1)}$ which comes with a sign of $(-1)^{n}$. These possibilities are pictured at the bottom of Figure 16 . Thus Subcase 4.2 contributes a factor of $2 s_{(n, n)}$ if $n$ is even and 0 if $n$ is odd.

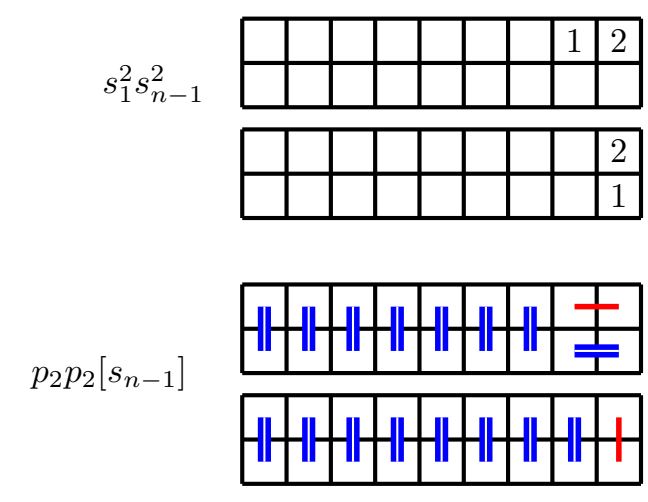

Figure 16. The diagrams for Case 4.2.

Subcase 4.3. $2 \leqslant a \leqslant n-1$. In this case we have four ways to obtain $s_{(a, 2 n-a)}$ from $s_{1}^{2} s_{n-1}^{2}$, namely, once from the product $s_{1}^{2} s_{(a-2,2 n-a)}$, once from the product $s_{1}^{2} s_{(a, 2 n-a-2)}$, and twice from the product $s_{1}^{2} s_{(a-1,2 n-a-1)}$. These possibilities are 
pictured at the top of Figure 17. There are two ways to obtain $s_{(a, 2 n-1)}$ from $p_{2} p_{2}\left[s_{n-1}\right]$, namely, once from the product $p_{2} s_{(a-2,2 n-a)}$ which comes with a sign of $(-1)^{a-2}$ and once from the product $p_{2} s_{(a, 2 n-a-2)}$ which comes with a sign of $(-1)^{a}$. These possibilities are pictured at the bottom of Figure 17. Thus Subcase 4.3 contributes a factor of

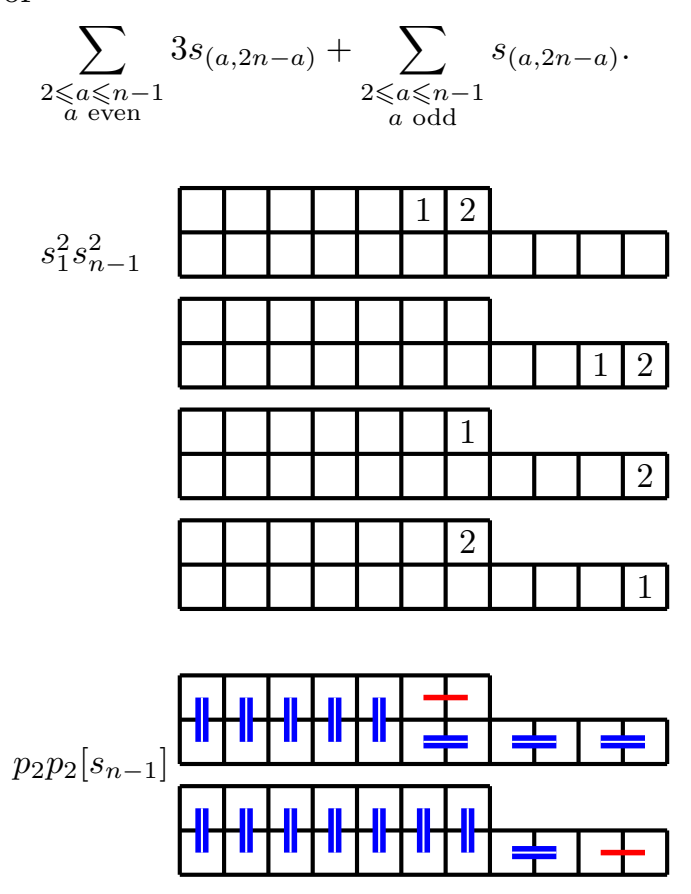

Figure 17. The diagrams for Case 4.3.

Thus the Schur functions in Case 4 contribute

to $s_{2}\left[s_{1} s_{n-1}\right]$.

$$
2 \chi(n \text { is even }) s_{(n, n)}+3 \sum_{\substack{2 \leqslant a \leqslant n-1 \\ a \text { even }}} s_{(a, 2 n-a)}+\sum_{\substack{1 \leqslant a \leqslant n-1 \\ a \text { odd }}} s_{(a, 2 n-a)}
$$

Case 5. $\lambda=(2 n)$. In this case, we have one way to obtain $s_{(2 n)}$ from $s_{1}^{2} s_{n-1}^{2}$, namely, it can arise in one way from the product $s_{1}^{2} s_{(2 n-2)}$. There also is only one way to obtain $s_{(2 n)}$ from $p_{2} p_{2}\left[s_{n-1}\right]$, namely, from the product $p_{2} s_{(2 n-2)}$ which comes with a sign of 1 . Thus Subcase 5 contributes a factor of $s_{(2 n)}$ to $s_{2}\left[s_{1} s_{n-1}\right]$.

Note that

$$
\begin{aligned}
s_{n}\left[s_{1} s_{1}\right] & =s_{n}\left[s_{2}+e_{2}\right] \\
& =\sum_{k=0}^{n} s_{k}\left[s_{2}\right] s_{n-k}\left[e_{2}\right] \\
& =\sum_{k=0}^{n}\left(\sum_{\substack{\lambda \text { even } \\
\lambda \vdash 2 k}} s_{\lambda}\right)\left(\sum_{\substack{\lambda^{\prime} \text { even } \\
\lambda \vdash 2(n-k)}} s_{\lambda}\right) .
\end{aligned}
$$

Our goal is to show that for all partitions of $2 n$ except $\lambda=(2,2 n-2)$,

$$
\left\langle s_{n}\left[s_{1} s_{1}\right], s_{\lambda}\right\rangle \geqslant\left\langle s_{2}\left[s_{1} s_{n-1}\right], s_{\lambda}\right\rangle \text {. }
$$


It follows from our previous theorem that the only $s_{\lambda}$ such that $\left\langle s_{2}\left[s_{1} s_{n-1}\right], s_{\lambda}\right\rangle \neq 0$ is when $\lambda$ is either contained in $(2,2 n, 2 n)$ or $\left(1^{2}, 2 n, 2 n\right)$. Now the only such $s_{\lambda}$ that appear in $\left(\sum_{\lambda \text { even, } \lambda \vdash 2 k} s_{\lambda}\right)$ are of the form

(1) $s_{(2, a, 2 k-a-2)}$ where $a$ is even and $2 \leqslant a \leqslant n-1$,

(2) $s_{(a, 2 k-a)}$ where $a$ is even and $2 \leqslant a \leqslant n$, or

(3) $s_{(2 k)}$.

The only such $s_{\lambda}$ that appear in $\left(\sum_{\lambda^{\prime} \text { even, } \lambda \vdash 2(n-k)} s_{\lambda}\right)$ are of the form

(1) $s_{\left(1^{2}, n-k-1, n-k-1\right)}$ or

(2) $s_{(n-k, n-k)}$.

Our goal now is to go through the five cases of the proof of Theorem 6.5 to show that in all but one case, $\left\langle s_{n}\left[s_{1} s_{1}\right], s_{\lambda}\right\rangle \geqslant\left\langle s_{2}\left[s_{1} s_{n-1}\right], s_{\lambda}\right\rangle$. However, we will need to apply the skew Schur function expansion rule of Remmel and Whitney [8]. Given a skew shape, $\lambda / \mu$ written in French notation, the reverse lexicographic filling of $\lambda / \mu$, $R L F(\lambda / \mu)$, is the filling of the Ferrers diagram with the integers $1, \ldots,|\lambda / \mu|$, which is obtained by filling the cells in order from right to left in each row, starting at the bottom row and proceeding upwards. For example, the $R L F((2,4,4,4) /(1,2))$ is pictured in Figure 18.

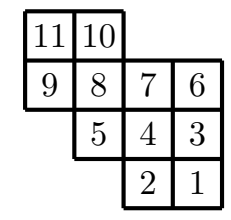

Figure 18. The reverse lexicographic filling of $(2,4,4,4) /(1,2)$.

A standard tableau $T$ of shape $\nu$ is said to $\lambda / \mu$-compatible if and only if $T$ satisfies the following two conditions.

(R1) If $x$ and $x+1$ lie in the same row in $R L F(\lambda / \mu)$, then $x+1$ appear strictly to the right and weakly below $x$ in $T$.

(R2) If $y$ is immediately above $x$ in $R L F(\lambda / \mu)$, then $y$ must appear strictly above and weakly to the left of $x$ in $T$.

A pictorial representation of these two conditions are depicted in Figure 19. We let $\lambda / \mu-\mathcal{S T}$ denote the set of all $\lambda / \mu$-compatible standard tableaux.

Then the skew Schur function expansion rule is that

$$
s_{\lambda / \mu}=\sum_{T \in \lambda / \mu-\mathcal{S T}} s_{s h(T)}
$$

where $\operatorname{sh}(T)$ is the shape of $T$. For example, if $\lambda=(2,2,3,4)$ and $\mu=(1,2,2)$, then in Figure 20, we have pictured the $R L F(\lambda / \mu)$ at the top right and we have constructed a tree which generates all $T$ in $\lambda / \mu-\mathcal{S T}$ by adding a new element at each stage in such a way that we obey rules $\mathrm{R} 1$ and $\mathrm{R} 2$. It follows that in this case

$$
s_{(2,2,3,4) /(1,2,2)}=s_{(1,1,2,2)}+s_{(1,1,1,3)}+s_{(2,2,2)}+2 s_{(1,2,3)}+s_{(1,1,4)}+s_{(3,3)}+s_{(2,4)} \text {. }
$$

Note that expanding a product of Schur functions is a special case of expanding a skew Schur function. That is, it easy to see that $s_{\lambda} s_{\mu}$ is equal to the skew Schur function $s_{\lambda * \mu}$ where $\lambda * \mu$ is the skew shape that arises by placing $\lambda$ above $\mu$ so that the bottom row of $\lambda$ starts just to the left of top row of $\mu$. For example, the if $\lambda=(2,2)$ and $\mu=(1,2)$, then $\lambda * \mu$ is pictured at the top-right of Figure 21 . The 
(R1)
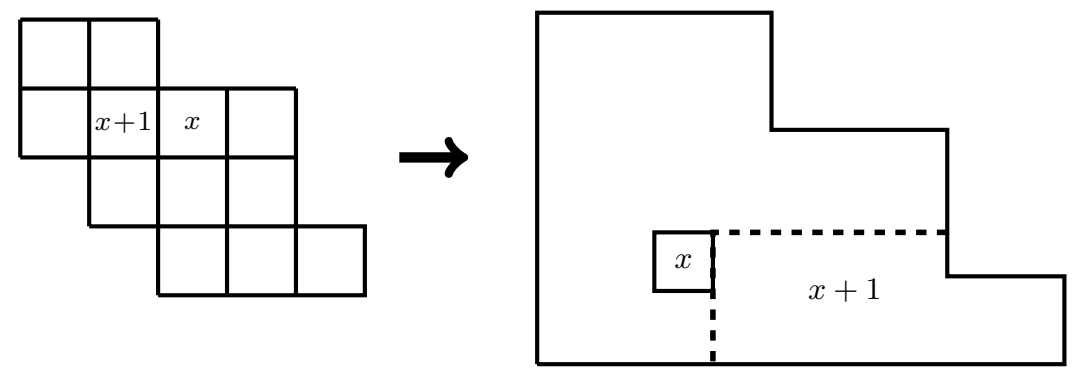

(R2)
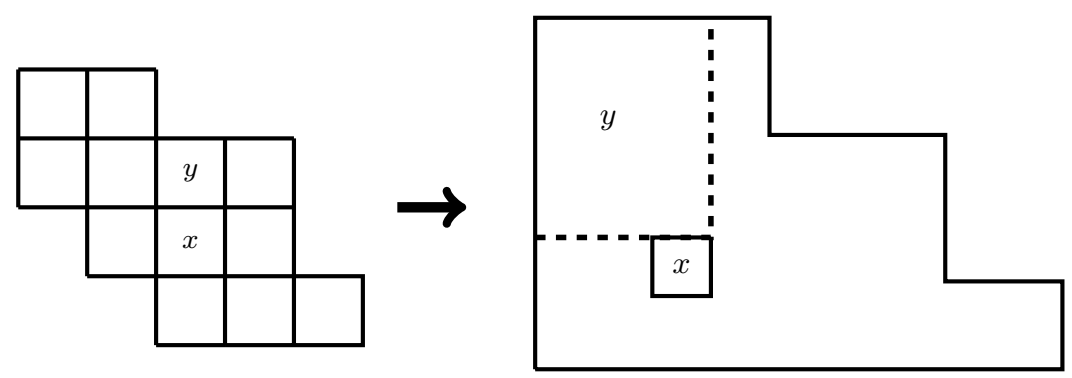

FiguRE 19. The two rules for $\lambda / \mu$-compatible standard tableaux.

other thing to observe is that if $\mu=\left(\mu_{1}, \ldots, \mu_{k}\right)$ where $0<\mu_{1} \leqslant \cdots \leqslant \mu_{k}$, then in the expansion of $s_{\lambda * \mu}$, the numbers in the part of the reverse lexicographic filling of $\mu$ are completely determined. That is, $1, \ldots, \mu_{k}$ must appear in row 1 , reading from left to right, the numbers $\mu_{k}+1, \ldots, \mu_{k}+\mu_{k-1}$ must appear in row 2 , reading from left to right, the numbers $\mu_{k}+\mu_{k-1}+1, \ldots, \mu_{k}+\mu_{k-1}+\mu_{k-2}$ must appear in row three, etc.. This means that in constructing our tree, we will eventually reach a single filling of shape $\mu$ and then we will continue to build our tree using the numbers of the reverse lexicographic filling that corresponding to $\lambda$. One can see an example of this phenomenon in Figure 20 in processing the numbers 1, 2, and 3 corresponding to the shape $(1,2)$. This means that we might as will ignore the numbers in $\mu$ and simply build our tree starting with a the Ferrers diagram of $\mu$ and using the numbers $1,2, \ldots,|\lambda|$ to fill the part corresponding to $\lambda$ in reverse lexicographic filling of $\lambda * \mu$. An example of this process is pictured in Figure 21 for the Schur function expansion of $s_{(2,2)} s_{(1,2)}$. In this case, we see that

$$
s_{(2,2)} s_{(1,2)}=s_{(1,2,2,2)}+s_{(1,1,2,3)}+s_{(2,2,3)}+s_{(1,3,3)}+s_{(1,2,4)}+s_{(3,4)} .
$$

We are ready to reanalyze the cases of the proof of Theorem 6.5 to show that in all but one case, $\left\langle s_{n}\left[s_{1} s_{1}\right], s_{\lambda}\right\rangle \geqslant\left\langle s_{2}\left[s_{1} s_{n-1}\right], s_{\lambda}\right\rangle$.

Case 1. $\lambda=\left(1^{2}, a, 2 n-2-a\right)$ where $a$ is odd and $1 \leqslant a \leqslant n-1$. In this case, we have shown that $\left\langle s_{2}\left[s_{1} s_{n-1}\right], s_{\left(1^{2}, a, 2 n-a-2\right)}\right\rangle=1$. It is easy see from the Pieri rule that $s_{\left(1^{2}, a, 2 n-a-2\right)}$ appears in the expansion of $s_{1^{4}} s_{(a-1,2 n-2-a-1)}$ with coefficient 1 . But the term $s_{\left(1^{4}\right)}$ appears in the expansion of $s_{2}\left[e_{2}\right]$ and the term $s_{(a-1,2 n-2-a-1)}$ appears in the expansion of $s_{n-2}\left[s_{2}\right]$ when $a$ is odd. Thus when $a$ is odd,

$$
\left\langle s_{n}\left[s_{1} s_{1}\right], s_{\left(1^{2}, a, 2 n-a-2\right)}\right\rangle \geqslant 1 \geqslant\left\langle s_{2}\left[s_{1} s_{n-1}\right], s_{\left(1^{2}, a, 2 n-a-2\right)}\right\rangle .
$$

Case 2. $\lambda=(2, a, 2 n-2-a)$ where $a$ is even and $2 \leqslant a \leqslant n-1$. In this case, we have shown that $\left\langle s_{2}\left[s_{1} s_{n-1}\right], s_{(2, a, 2 n-a-2)}\right\rangle=1$. Since $(2, a, 2 n-a-2)$ has even parts, 


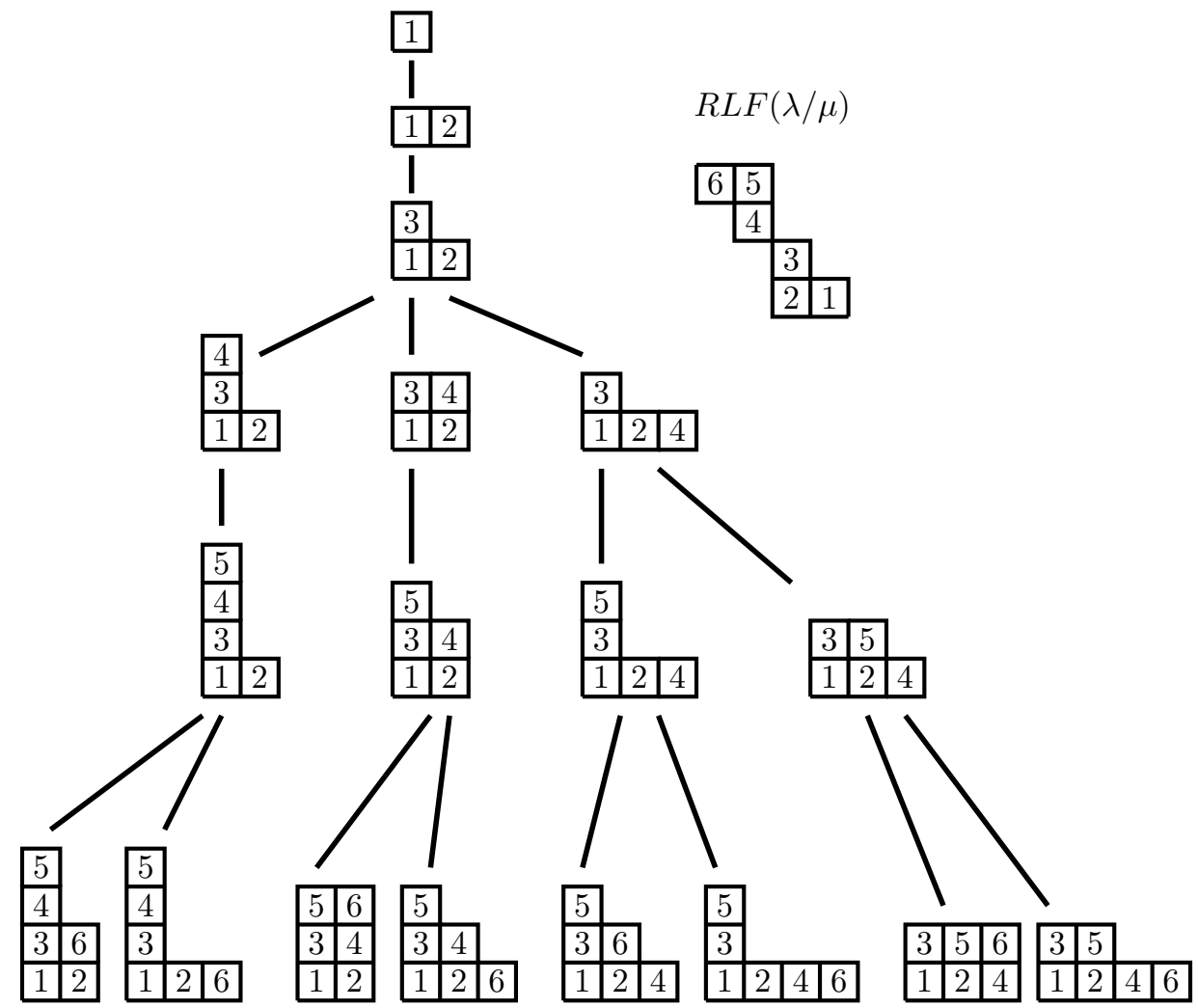

FIGURE 20. The tree for the expansion of $s_{(2,2,3,4) /(1,2,2)}$.

$s_{(2, a, 2 n-a-2)}$ appears in the expansion of $s_{n}\left[s_{2}\right]$ so that

$$
\left\langle s_{n}\left[s_{1} s_{1}\right], s_{(2, a, 2 n-a-2)}\right\rangle \geqslant 1 \geqslant\left\langle s_{2}\left[s_{1} s_{n-1}\right], s_{(2, a, 2 n-a-2)}\right\rangle .
$$

Case 3. $\lambda=(1, a, 2 n-1-a)$ where $1 \leqslant a \leqslant n-1$. First we consider the case where $a=1$. In this case, we have shown that $\left\langle s_{2}\left[s_{1} s_{n-1}\right], s_{\left(1^{2}, 2 n-2\right)}\right\rangle=1$. It is easy see from the Pieri rule that $s_{\left(1^{2}, 2 n-2\right)}$ appears in the expansion of $s_{1^{2}} s_{(2 n-2)}$ with coefficient 1. But the term $s_{1^{2}}$ appears in the expansion of $s_{1}\left[e_{2}\right]$ and the term $s_{(2 n-2)}$ appears in the expansion of $s_{n-1}\left[s_{2}\right]$. Thus

$$
\left\langle s_{n}\left[s_{1} s_{1}\right], s_{\left(1^{2}, 2 n-2\right)}\right\rangle \geqslant 1 \geqslant\left\langle s_{2}\left[s_{1} s_{n-1}\right], s_{\left(1^{2}, 2 n-2\right)}\right\rangle .
$$

When $2 \leqslant a \leqslant n-1$, we have shown that $\left\langle s_{2}\left[s_{1} s_{n-1}\right], s_{(1, a, 2 n-1-a)}\right\rangle=2$. The first thing to observe is that when $a$ is even, then $s_{(a, 2 n-2-a)}$ appears in the expansion of $s_{n-1}\left[s_{2}\right]$ from which it follows that $s_{(1, a, 2 n-1-a)}$ will occur with multiplicity 1 in the Schur function expansion of $s_{1}\left[e_{2}\right] s_{n-1}\left[s_{2}\right]=s_{\left(1^{2}\right)} s_{n-1}\left[s_{2}\right]$. Similarly, when $a$ is odd, then $s_{(a-1,2 n-1-a)}$ appears in the expansion of $s_{n-1}\left[s_{2}\right]$ from which it follows that $s_{(1, a, 2 n-1-a)}$ will occur with multiplicity 1 in the Schur function expansion of $s_{1}\left[e_{2}\right] s_{n-1}\left[s_{2}\right]=s_{\left(1^{2}\right)} s_{n-1}\left[s_{2}\right]$. This situation is pictured at the top of Figure 22. If $2 \leqslant a \leqslant n-1$, then $s_{(1, a, 2 n-1-a)}$ will occur in the expansion of $s_{(2,2)} s_{n-2}\left[s_{2}\right]$ as is evidenced by the pictures in the middle row of Figure 22. Note that in the case where $a=n-1$ and $a$ is odd, the expansion still works. It will just be the case that the 4 is on top of the 1 . Hence in this case we get another occurrence of $s_{(1, a, 2 n-1-a)}$ from the term $s_{2}\left[e_{2}\right] s_{n-2}\left[s_{2}\right]$. This will show that for $2 \leqslant a \leqslant n-1$,

$$
\left\langle s_{n}\left[s_{1} s_{1}\right], s_{(1, a, 2 n-1-a)}\right\rangle \geqslant 2 \geqslant\left\langle s_{2}\left[s_{1} s_{n-1}\right], s_{\left(1^{2}, 2 n-2\right)}\right\rangle .
$$




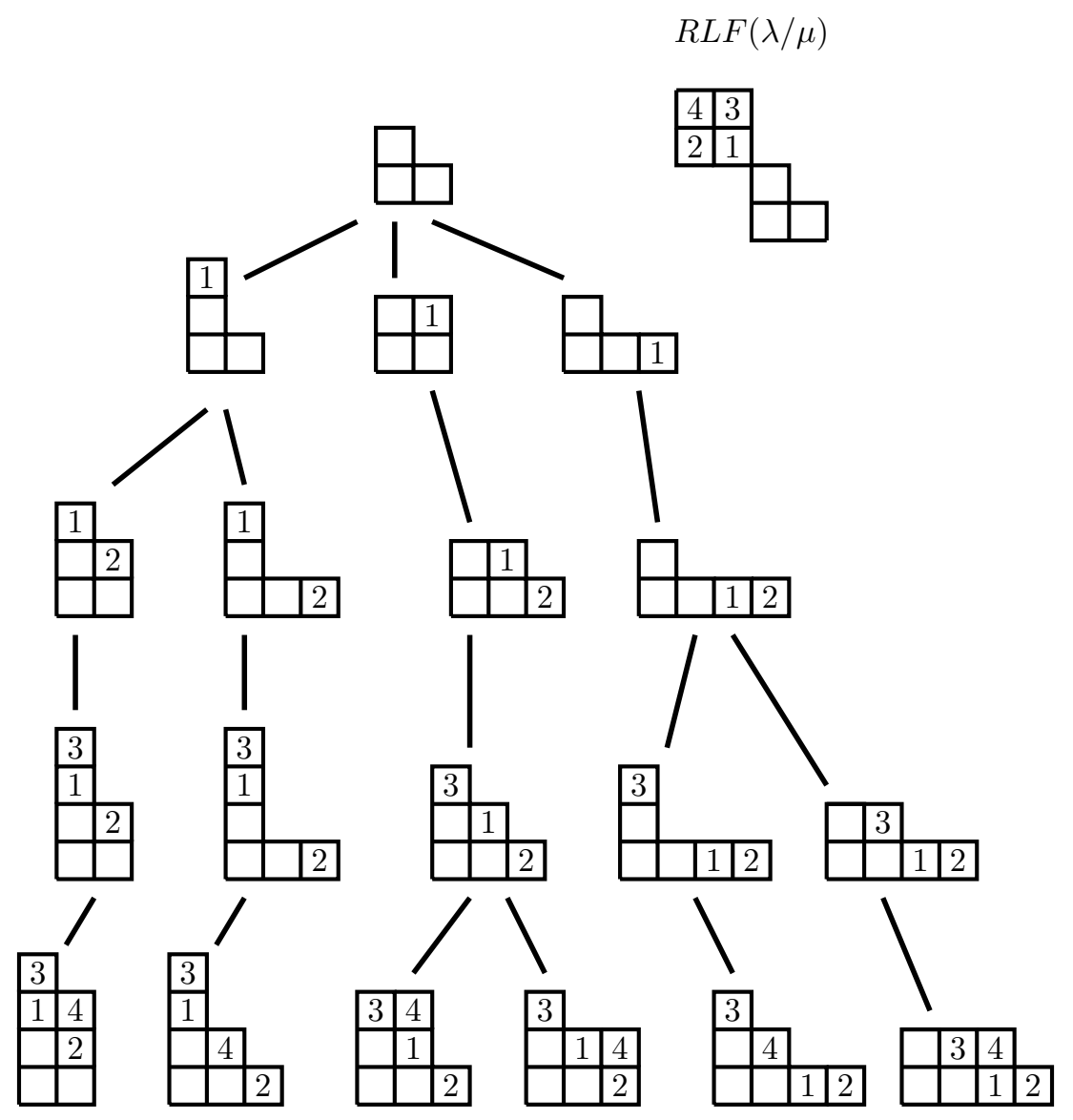

Figure 21. The tree for the expansion of $s_{(2,2)} s_{(1,2)}$.

In fact, in the case where $a=n-1$, we note that $s_{(n-1, n-1)}$ appears in the expansion of $s_{n-1}\left[e_{2}\right]$ which implies that we would get another copy of $s_{(1, n-1, n)}$ in the expansion of $s_{1}\left[s_{2}\right] s_{n-1}\left[e_{2}\right]=s_{2} s_{n-1}\left[e_{2}\right]$ as pictured at the bottom of Figure 22 .

Case 4. $\lambda=(a, 2 n-a)$. In this case, we can give an explicit formula for $\left\langle s_{n}\left[s_{1} s_{1}\right]\right.$, $\left.s_{(a, 2 n-a)}\right\rangle$. The key observation to make is that the only Schur function $s_{\lambda}$ which appears in the expansion of $s_{n-k}\left[e_{2}\right]$ where $\lambda$ is a 2-part partition is $s_{(n-k, n-k)}$. In addition, it is easy to see from our Skew Schur function expansion rule that the only $s_{\lambda}$ which appears in the expansion of a product $s_{(n-k, n-k)} s_{(a, b)}$ where $\lambda$ is a 2-part partition is $s_{(n-k+a, n-k+b)}$. That is, one can see that when multiplying a Schur function $s_{(a, b)}$ by any Schur function $s_{(c, c)}$, the only way to get a shape with two parts is to put all the elements in first row of the reverse lexicographic filling corresponding to $(c, c)$ in the first row and all the elements in the second row of the reverse lexicographic filling corresponding to $(c, c)$ in the second row. This process is pictured in Figure 23. 
$a$ odd

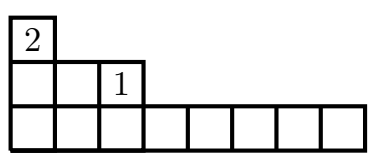

3

$s_{\left(2^{2}\right)} s_{n-1}\left[s_{2}\right]$
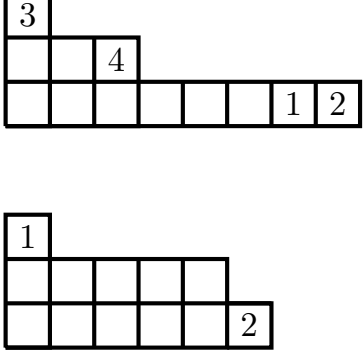

$a$ even
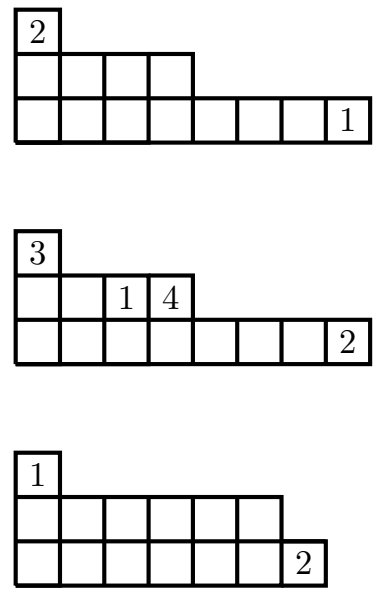

Figure 22. Possibilities for $s_{(1, a, 2 n-1-a)}$ in the expansion of $s_{n}\left[s_{1} s_{1}\right]$.

Reverse lexicographic filling
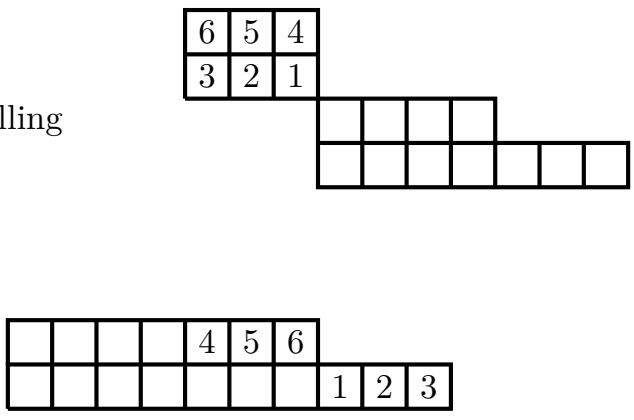

FigURE 23. Mutliplying $s_{(a, b)}$ by $s_{(c, c)}$.

It follows that if $\lambda=(a, 2 n-a)$, then we have

$$
\begin{aligned}
\left\langle\sum_{k=0}^{n} s_{k}\left[s_{2}\right] s_{n-k}\left[e_{2}\right], s_{(a, 2 n-a)}\right\rangle & \\
& =\left\langle\sum_{k=0}^{n} s_{k}\left[s_{2}\right] s_{(n-k, n-k)}, s_{(a, 2 n-a)}\right\rangle \\
& =\sum_{k=0}^{n} \chi(n-k<a \text { and }(a-(n-k), 2 n-a-(n-k)) \text { is even). }
\end{aligned}
$$

Thus

$$
\left\langle\sum_{k=0}^{n} s_{k}\left[s_{2}\right] s_{n-k}\left[e_{2}\right], s_{(a, 2 n-a)}\right\rangle= \begin{cases}\frac{a}{2}+1 & \text { if } a \text { is even; } \\ \frac{a+1}{2} & \text { if } a \text { is odd. }\end{cases}
$$

It follows that if $a$ is odd and $1 \leqslant a \leqslant n-1$, then

$$
\left\langle s_{n}\left[s_{1} s_{1}\right], s_{(a, 2 n-a)}\right\rangle \geqslant 1=\left\langle s_{2}\left[s_{1} s_{n-1}\right], s_{(a, 2 n-a)}\right\rangle .
$$


If $a$ is even and $4 \leqslant a \leqslant n-1$, then

$$
\left\langle s_{n}\left[s_{1} s_{1}\right], s_{(a, 2 n-a)}\right\rangle \geqslant 3=\left\langle s_{2}\left[s_{1} s_{n-1}\right], s_{(a, 2 n-a)}\right\rangle .
$$

However, if $a=2$, then $\left\langle s_{n}\left[s_{1} s_{1}\right], s_{(a, 2 n-a)}\right\rangle=2$ while $\left\langle s_{2}\left[s_{1} s_{n-1}\right], s_{(a, 2 n-a)}\right\rangle=3$ so we do not have

$$
\left\langle s_{n}\left[s_{1} s_{1}\right], s_{(2,2 n-2)}\right\rangle \geqslant\left\langle s_{2}\left[s_{1} s_{n-1}\right], s_{(2,2 n-2)}\right\rangle .
$$

The only other Schur function corresponding to a two row shape that we have not considered is $s_{(n, n)}$. In this case,

$$
\left\langle s_{2}\left[s_{1} s_{n-1}\right], s_{(n, n)}\right\rangle= \begin{cases}0 & \text { if } a \text { is odd; } \\ 2 & \text { if } a \text { is even. }\end{cases}
$$

Note however, if $n$ is even, then $s_{(n, n)}$ occurs in the expansion of $s_{n}\left[s_{2}\right]$ and the expansion of $s_{n}\left[e_{2}\right]$ so that

$$
\left\langle s_{n}\left[s_{1} s_{1}\right], s_{(n, n)}\right\rangle \geqslant 2=\left\langle s_{2}\left[s_{1} s_{n-1}\right], s_{(n, n)}\right\rangle .
$$

Case 5. $\lambda=(2 n)$. In this case, we proved that $\left\langle s_{2}\left[s_{1} s_{n-1}\right], s_{(2 n)}\right\rangle=1$. But $s_{(2 n)}$ clearly appears in the expansion of $s_{n}\left[s_{2}\right]$ so that

$$
\left\langle s_{n}\left[s_{1} s_{1}\right], s_{(2 n)}\right\rangle \geqslant\left\langle s_{2}\left[s_{1} s_{n-1}\right], s_{(2 n)}\right\rangle .
$$

Acknowledgements. We thank the anonymous referee for their comments and suggestions which improved the quality of our paper drastically.

\section{REFERENCES}

[1] Mahir Bilen Can, A representation on labeled rooted forests, Commun. Algebra 46 (2018), no. 10 , 4273-4291.

[2] Yao Min Chen, Adriano M. Garsia, and Jeffrey Remmel, Algorithms for plethysm, in Combinatorics and algebra (Boulder, Colo., 1983), Contemporary Mathematics, vol. 34, American Mathematical Society, 1984, pp. 109-153.

[3] Louis Comtet, Advanced combinatorics. The art of finite and infinite expansions, enlarged ed., Reidel Publishing Co., 1974.

[4] Olexandr Ganyushkin and Volodymyr Mazorchuk, Classical finite transformation semigroups: an introduction, Algebra and Applications, vol. 9, Springer, 2009.

[5] Dudley E. Littlewood, The Theory of Group Characters and Matrix Representations of Groups, Oxford University Press, 1940.

[6] Nicholas A. Loehr and Jeffrey Remmel, A computational and combinatorial exposé of plethystic calculus, J. Algebr. Comb. 33 (2011), no. 2, 163-198.

[7] Ian Grant Macdonald, Symmetric functions and Hall polynomials, second ed., Oxford Mathematical Monographs, Clarendon Press, 1995.

[8] Jeffrey Remmel and Roger Whitney, Multiplying Schur functions, J. Algorithms 5 (1984), no. 4 471-487.

[9] Robert M. Thrall, On symmetrized Kronecker powers and the structure of the free Lie ring, Am. J. Math. 64 (1942), 371-388.

Mahir Bilen Can, Tulane University, Mathematics Department, New Orleans, USA

E-mail : mahirbilencan@gmail.com

Jeff RemmeL ${ }^{\dagger}$, UC San Diego, Mathematics Department, La Jolla, USA 